Supporting Information: Madurastatin D1 and D2, Oxazoline Containing Siderophores Isolated from an Actinomadura sp.

\title{
Madurastatin D1 and D2, Oxazoline Containing Siderophores Isolated from an Actinomadura sp.
}

Jia-Xuan Yan , ${ }^{1}$ Marc G. Chevrette, ${ }^{2,4}$ Doug R. Braun, ${ }^{1}$ Mary Kay Harper, ${ }^{3}$ Cameron R. Currie $^{4}$ and Tim S. Bugni ${ }^{1, *}$

1 Pharmaceutical Sciences Division, University of Wisconsin-Madison, 777 Highland Ave, Madison, WI, 53705, USA;

2 Department of Genetics, University of Wisconsin-Madison, 425 G Henry Mall, Madison, WI, 53706, USA;

3 Department of Medicinal Chemistry, University of Utah, 30 South 2000 East, Salt Lake City, UT, 84112, USA;

${ }^{4}$ Department of Bacteriology, University of Wisconsin-Madison, 1550 Linden Ave, Madison, WI, 53706, USA;

* To whom correspondence should be addressed: E-mail: tim.bugni@wisc.edu; Tel.: +1-608-263-2519; Fax: +1-608-262-5345 
1. General Experimental Procedures and References

2. Figure S1. ${ }^{1} \mathrm{H}$ NMR spectrum of (-)-madurastatin $\mathrm{C} 1 \quad(1,400 \mathrm{MHz}$,

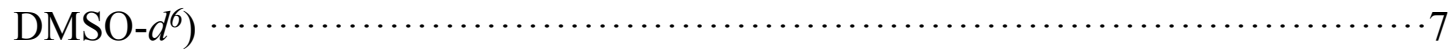

3. Figure S2. ${ }^{13} \mathrm{C}$ NMR spectrum of (-)-madurastatin $\mathrm{C} 1 \quad(1,101 \mathrm{MHz}$,

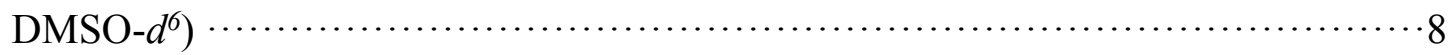

4. Figure S3. ${ }^{1} \mathrm{H}$ NMR spectrum of madurastatin D1 (2, $\left.500 \mathrm{MHz}, \mathrm{DMSO}-d^{6}\right) \cdots \cdots \cdot 9$

5. Figure S4. ${ }^{13} \mathrm{C}$ NMR spectrum of madurastatin D1 (2, $\left.125 \mathrm{MHz}, \mathrm{DMSO}-d^{6}\right) \cdots \cdots$

6. Figure S5. gCOSY spectrum of madurastatin D1 (2, $\left.600 \mathrm{MHz}, \mathrm{DMSO}-d^{6}\right) \cdots \cdots 11$

7. Figure S6. gHSQC spectrum of madurastatin D1 (2, $\left.600 \mathrm{MHz}, \mathrm{DMSO}-d^{6}\right) \cdots \cdots \cdot 12$

8. Figure S7. gHMBC spectrum of madurastatin D1 (2, $\left.600 \mathrm{MHz}, \mathrm{DMSO}-d^{6}\right) \cdots \cdots 13$

9. Figure S8. ${ }^{1} \mathrm{H}$ NMR spectrum of madurastatin D2 (3, $\left.500 \mathrm{MHz}, \mathrm{DMSO}-d^{6}\right) \cdots \cdots 14$

10. Figure S9. ${ }^{13} \mathrm{C}$ NMR spectrum of madurastatin D2 (3, $\left.125 \mathrm{MHz}, \mathrm{DMSO}-d^{6}\right) \cdots 15$

11. Figure S10. gCOSY spectrum of madurastatin D2 (3, $\left.600 \mathrm{MHz}, \mathrm{DMSO}-d^{6}\right) \cdots 16$

12. Figure S11. gHSQC spectrum of madurastatin D2 (3, $\left.600 \mathrm{MHz}, \mathrm{DMSO}-d^{6}\right) \cdots 17$

13. Figure S12. gHMBC spectrum of madurastatin D2 (3, $\left.600 \mathrm{MHz}, \mathrm{DMSO}-d^{6}\right) \cdots 18$

14. Figure S13. Positive Ion HRESIMS of (-)-madurastatin $\mathrm{C} 1$ (1) and the Fe

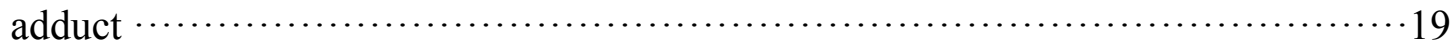

15. Figure S14. Positive ion HRESIMS of madurastatin D1 (2) and the Fe

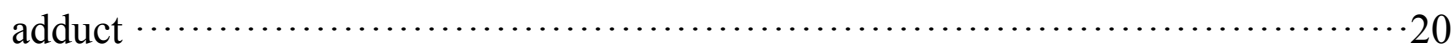

16. Figure S15. Positive ion HRESIMS of madurastatin D1 (3) and the Fe

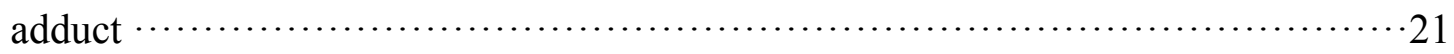

17. Figure S16. Chrome Azurol S (CAS) assay of compounds 1$3 \cdots \cdots \cdots \cdots \cdot \cdots \cdot 22$

18. Figure S17. LCMS spectra of Marfey's analysis of (-)-madurastatin C1 (1) $\cdots \cdot 23$

19. Table S1. DFT calculated ${ }^{13} \mathrm{C}$ NMR Chemical Shifts of

madurastatin D1 (2) diastereoisomers models...

20. Table S2. DP4 probability calculation of madurastatin D1 (2) diastereoisomer

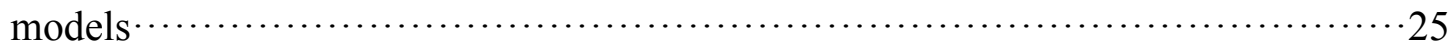

21. Table S3. Annotations for the mad biosynthetic gene cluster $\cdots \cdots \cdots \cdots \cdots \cdot 26-27$ 


\section{General Experimental Procedures}

Optical rotations were measured on a Perkin-Elmer 241 Polarimeter. UV spectra were recorded on an Aminco/OLIS UV-Vis spectrophotometer. IR spectra were measured with a Bruker Equinox 55/S FT-IR spectrophotometer. NMR spectra were obtained in $d_{6}$-DMSO ( $\left.\delta_{H} 2.50 \mathrm{ppm}, \delta_{C} 39.50 \mathrm{ppm}\right)$ with a Bruker Avance 600 III $\mathrm{MHz}$ spectrometer equipped with a ${ }^{1} \mathrm{H}\left\{{ }^{13} \mathrm{C} /{ }^{15} \mathrm{~N} /{ }^{31} \mathrm{P}\right\}$ cryoprobe, a Bruker Avance III $500 \mathrm{MHz}$ spectrometer equipped with a ${ }^{13} \mathrm{C} /{ }^{15} \mathrm{~N}\left\{{ }^{1} \mathrm{H}\right\}$ cryoprobe, and a Bruker Avance III HD $400 \mathrm{MHz}$ spectrometer. HRMS data were acquired with a Bruker MaXis ${ }^{\mathrm{TM}} 4 \mathrm{G}$ ESI-QTOF mass spectrometer. RP HPLC was performed using a Shimadzu Prominence HPLC system and a Phenomenex Luna phenyl-hexyl column $(250 \times 4.6$ $\mathrm{mm}$ ). UHPLC-HRMS was acquired using a Bruker MaXis ${ }^{\mathrm{TM}}$ 4G ESI-QTOF mass spectrometer coupled with a Waters Acquity UPLC system operated by Bruker Hystar software and a C18 column (Phenomenex Kinetex $2.6 \mu \mathrm{m}, 2.1 \mathrm{~mm} \times 100 \mathrm{~mm}$ ).

\section{Biological Material}

Sponge specimens were collected on August 7, 2013 near Stan Blum State Park boat launch $\left(27^{\circ} 49^{\prime} 45.7^{\prime} \mathrm{N}, 80^{\circ} 18^{\prime} 42.8^{\prime \prime} \mathrm{W}\right)$ in Florida. A voucher specimen is housed at the University of Wisconsin-Madison. For cultivation, a sample of sponge $\left(1 \mathrm{~cm}^{3}\right)$ was ground in $500 \mu \mathrm{L}$ sterile seawater and dilutions were made using $500 \mu \mathrm{L}$ sterile seawater. Subsequently, $400 \mu \mathrm{L}$ of diluted sponge sample was added to $200 \mu \mathrm{L}$ of sterile seawater and $100 \mu \mathrm{L}$ was plated using a sterile L-shaped spreader. Diluted sample was plated on $\mathrm{R}^{2} \mathrm{~A}^{1}$ media supplemented with artificial seawater. Each medium was supplemented with $50 \mu \mathrm{g} / \mathrm{mL}$ cycloheximide, $25 \mu \mathrm{g} / \mathrm{mL}$ nystatin, and 25 $\mu \mathrm{g} / \mathrm{mL}$ nalidixic acid. Plates were incubated at $28^{\circ} \mathrm{C}$ and colonies were isolated over the course of two months.

\section{Sequencing}

16S rDNA sequencing was conducted as previously described ${ }^{2}$. WMMA-1423 was identified as an Actinomadura sp. The 16S sequence for WMMA-1423 was deposited in GenBank (accession number KY014999).

\section{Fermentation, Extraction and Isolation}

Two $10 \mathrm{~mL}$ seed cultures $(25 \times 150 \mathrm{~mm}$ tubes $)$ in medium DSC $(20 \mathrm{~g}$ soluble starch, $10 \mathrm{~g}$ glucose, $5 \mathrm{~g}$ peptone, $5 \mathrm{~g}$ yeast extract per liter of artificial seawater) were inoculated with strain WMMA-1423 and shaken (200 RPM, $28{ }^{\circ} \mathrm{C}$ ) for seven days. Two liter flask $(1 \times 500 \mathrm{~mL})$ containing ASW-A $(20 \mathrm{~g}$ soluble starch, $10 \mathrm{~g}$ glucose, 5 g peptone, $5 \mathrm{~g}$ yeast extract, $5 \mathrm{~g} \mathrm{CaCO}_{3}$ per liter of artificial seawater) were inoculated with $20 \mathrm{~mL}$ seed culture and were incubated (200 RPM, $\left.28{ }^{\circ} \mathrm{C}\right)$ for seven days. Four-liter flasks $(6 \times 1 \mathrm{~L})$ containing medium RAM2 $(4 \mathrm{~g}$ corn meal, $10 \mathrm{~g}$ glucose, 15 $\mathrm{g}$ maltose, $7.5 \mathrm{~g}$ pharmamedia, $5 \mathrm{~g}$ yeast extract per liter of 1:1 artificial seawater and distilled water) with Diaion HP20 (7\% by weight) were inoculated with $50 \mathrm{~mL}$ from the $500 \mathrm{~mL}$ culture and shaken (200 RPM, $28{ }^{\circ} \mathrm{C}$ ) for seven days. For making artificial sea water, solutions I (415.2 g NaCl, $69.54 \mathrm{~g} \mathrm{Na}_{2} \mathrm{SO}_{4}, 11.74 \mathrm{~g} \mathrm{KCl}, 3.40 \mathrm{~g}$ $\left.\mathrm{NaHCO}_{3}, 1.7 \mathrm{~g} \mathrm{KBr}, 0.45 \mathrm{~g} \mathrm{H}_{3} \mathrm{BO}_{3}, 0.054 \mathrm{~g} \mathrm{NaF}\right)$ and II (187.9 g MgCl $2 \cdot 6 \mathrm{H}_{2} \mathrm{O}, 22.72$ $\mathrm{g} \mathrm{CaCl} 2 \cdot 2 \mathrm{H}_{2} \mathrm{O}, 0.428 \mathrm{~g} \mathrm{SrCl}_{2} \cdot 6 \mathrm{H}_{2} \mathrm{O}$ ) were made up separately using distilled water and combined to give a total volume of $20 \mathrm{~L}$.

Filtered HP20 and cells were washed with $\mathrm{H}_{2} \mathrm{O}$ and extracted with acetone. The 
acetone extract was subjected to liquid-liquid partitioning using 30\% aqueous $\mathrm{MeOH}$ and $\mathrm{CHCl}_{3}(1: 1)$. The $\mathrm{CHCl}_{3}$-soluble partition (1.59 g) was fractionated by Sephadex LH20 column chromatography (column size $500 \times 40 \mathrm{~mm}, \mathrm{CHCl}_{3}: \mathrm{MeOH}=1: 1,20 \mathrm{~mL}$ for each fraction). Fractions containing 2 and $\mathbf{3}(157.2 \mathrm{mg})$ were further fractionated using a benchtop HP20-ss column with a gradient from $10 \% / 90 \% \mathrm{MeOH} / \mathrm{H}_{2} \mathrm{O}$ to $100 \% \mathrm{MeOH}$ (column size $500 \times 40 \mathrm{~mm}, 100 \mathrm{~mL}$ eluent for each fraction). The $60 \% / 40 \% \mathrm{MeOH} / \mathrm{H}_{2} \mathrm{O}$ fraction $(26.3 \mathrm{mg})$ was subjected to RP HPLC $(10 \% / 90 \%$ to $40 \% / 60 \% \mathrm{MeCN} / \mathrm{H}_{2} \mathrm{O}, 39.5 \mathrm{~min}, 4 \mathrm{~mL} / \mathrm{min}$ ) using a Phenomenex Luna phenyl hexyl column $(250 \times 4.6 \mathrm{~mm})$, yielding $1\left(15.2 \mathrm{mg}, t_{R} 22.5 \mathrm{~min}\right)$. The $100 \% \mathrm{MeOH}$ fraction (46.2 $\mathrm{mg}$ ) was subjected to RP HPLC $\left(20 \% / 80 \%\right.$ to $50 \% / 50 \% \mathrm{MeCN} / \mathrm{H}_{2} \mathrm{O}, 39.5 \mathrm{~min}$, $4 \mathrm{~mL} / \mathrm{min})$ using a Phenomenex Luna phenyl hexyl column $(250 \times 4.6 \mathrm{~mm})$, yielding $2\left(1.1 \mathrm{mg}, t_{R} 16.2 \mathrm{~min}\right)$ and $\mathbf{3}\left(3.9 \mathrm{mg}, t_{R} 21.0 \mathrm{~min}\right)$.

(-)-Madurastatin C1 (1): light yellow oil, $[\alpha]^{25}{ }_{436}=-16.8$ (c 2.5, MeOH); HRMS $[\mathrm{M}+\mathrm{H}]^{+} m / z=592.2735$ (calcd. for $\mathrm{C}_{26} \mathrm{H}_{38} \mathrm{~N}_{7} \mathrm{O}_{9}{ }^{+}$592.2726), $[\mathrm{M}-2 \mathrm{H}+\mathrm{Fe}]^{+} m / z=$ 645.1841 (calcd. for $\mathrm{C}_{26} \mathrm{H}_{35} \mathrm{~N}_{7} \mathrm{O}_{9} \mathrm{Fe}^{+}$645.1829). ${ }^{1} \mathrm{H}$ NMR (400 MHz, DMSO- $\left.d_{6}\right) \delta$ $8.49(\mathrm{t}, J=5.8 \mathrm{~Hz}, 1 \mathrm{H}), 8.17(\mathrm{~d}, J=8.2 \mathrm{~Hz}, 1 \mathrm{H}), 7.92$ (t, $J=5.4 \mathrm{~Hz}, 1 \mathrm{H}), 7.64(\mathrm{dd}, J$ $=7.8,1.6 \mathrm{~Hz}, 1 \mathrm{H}), 7.53-7.40(\mathrm{~m}, 1 \mathrm{H}), 7.00(\mathrm{~d}, J=8.2 \mathrm{~Hz}, 1 \mathrm{H}), 6.94(\mathrm{dd}, J=11.5$, $4.2 \mathrm{~Hz}, 1 \mathrm{H}), 5.01(\mathrm{dd}, J=10.4,7.8 \mathrm{~Hz}, 1 \mathrm{H}), 4.64(\mathrm{dd}, J=10.3,8.5 \mathrm{~Hz}, 1 \mathrm{H}), 4.52(\mathrm{t}, J$ $=8.1 \mathrm{~Hz}, 1 \mathrm{H}), 4.37-4.27(\mathrm{~m}, 1 \mathrm{H}), 3.81-3.62(\mathrm{~m}, 4 \mathrm{H}), 3.26(\mathrm{~d}, J=6.1 \mathrm{~Hz}, 2 \mathrm{H})$, $2.93(\mathrm{t}, J=6.3 \mathrm{~Hz}, 1 \mathrm{H}), 2.56-2.51(\mathrm{~m}, 2 \mathrm{H}), 2.23(\mathrm{~s}, 3 \mathrm{H}), 1.95-1.82(\mathrm{~m}, 4 \mathrm{H}), 1.72-$ $1.38(\mathrm{~m}, 5 \mathrm{H}) .{ }^{13} \mathrm{C}$ NMR $\left(101 \mathrm{MHz}, \mathrm{DMSO}-d_{6}\right) \delta 173.2,171.1,170.3,168.5,166.0$, 165.0, 159.1, 134.2, 128.2, 119.2, 116.7, 110.0, 69.5, 67.5, 63.5, 51.3, 49.6, 47.1, 42.3, $34.7,34.0,32.0,30.0,27.8,22.8,20.4$.

Madurastatin D1 (2): light yellow oil, $[\alpha]^{25}{ }_{436}=+16.6$ (c $\left.0.6, \mathrm{MeOH}\right)$; UV-Vis $(\mathrm{MeOH}): \lambda_{\max }(\log \varepsilon) 224 \mathrm{~nm}$ (3.03), $243 \mathrm{~nm}$ (2.98), $306 \mathrm{~nm}$ (2.71); IR (ATR): $v_{\max }$ 3329, 2944, 2832, 1639, 1547, 1530, 1449, 1413, 1330, 1258, 1233, 1200, 1114, 1023, 922, 826, 759, $667 \mathrm{~cm}^{-1}$; HRMS [M+H] $]^{+} m / z=618.2876$ (calcd. for $\mathrm{C}_{28} \mathrm{H}_{40} \mathrm{~N}_{7} \mathrm{O}_{9}{ }^{+}$ 618.2882), [M-2H+Fe] ${ }^{+} m / z=671.1988$ (calcd. for $\mathrm{C}_{28} \mathrm{H}_{37} \mathrm{~N}_{7} \mathrm{O}_{9}{ }^{+}$671.1986). ${ }^{1} \mathrm{H}$ NMR $\left(500 \mathrm{MHz}, \mathrm{DMSO}-d_{6}\right) \delta 8.61(\mathrm{t}, J=5.9 \mathrm{~Hz}, 1 \mathrm{H}), 8.01(\mathrm{t}, J=5.4 \mathrm{~Hz}, 1 \mathrm{H}), 7.64(\mathrm{dd}, J$ $=7.6,1.4 \mathrm{~Hz}, 1 \mathrm{H}), 7.47(\mathrm{ddd}, J=8.4,7.3,1.6 \mathrm{~Hz}, 1 \mathrm{H}), 7.01(\mathrm{~d}, J=8.4 \mathrm{~Hz}, 1 \mathrm{H}), 6.95$ $(\mathrm{t}, J=7.5 \mathrm{~Hz}, 1 \mathrm{H}), 5.01((\mathrm{dd}, J=10.4,7.7 \mathrm{~Hz}, 1 \mathrm{H}), 4.65(\mathrm{dd}, J=10.3,8.5 \mathrm{~Hz}, 1 \mathrm{H})$, $4.51(\mathrm{t}, J=8.0 \mathrm{~Hz}, 1 \mathrm{H}), 4.32-4.24(\mathrm{~m}, 1 \mathrm{H}), 4.01(\mathrm{q}, J=5.0 \mathrm{~Hz}, 1 \mathrm{H}), 3.73(\mathrm{dd}, J=$ $16.6,6.0 \mathrm{~Hz}, 1 \mathrm{H}), 3.67(\mathrm{dd}, J=16.6,6.0 \mathrm{~Hz}, 1 \mathrm{H}), 3.57-3.41(\mathrm{~m}, 2 \mathrm{H}), 3.24(\mathrm{dd}, J=$ 6.6, 6.0 Hz, 2H), $2.88(\mathrm{~m}, 1 \mathrm{H}), 2.56-2.48(\mathrm{~m}, 2 \mathrm{H}), 2.29(\mathrm{~s}, 3 \mathrm{H}), 2.03-1.76(\mathrm{~m}, 6 \mathrm{H})$, $1.70-1.43(\mathrm{~m}, 2 \mathrm{H}), 1.21(\mathrm{~d}, J=5.2 \mathrm{~Hz}, 3 \mathrm{H}) .{ }^{13} \mathrm{C} \mathrm{NMR}\left(125 \mathrm{MHz}, \mathrm{DMSO}-d_{6}\right) \delta$ 171.6, 170.8, 170.2, 168.4, 165.9, 162.4, 159.1, 134.1, 128.1, 119.1, 116.7, 110.0, 74.7, 69.5, 67.5, 64.8, 51.8, 51.1, 47.4, 42.2, 37.9, 34.7, 31.9, 26.6, 21.6, 21.6, 20.9, 19.7 .

Madurastatin D2 (3): light yellow oil, $[\alpha]^{25}{ }_{436}=-184.3(c$ 1.4, MeOH); UV-Vis $(\mathrm{MeOH}): \lambda_{\max }(\log \varepsilon) 224 \mathrm{~nm}$ (3.79), $243 \mathrm{~nm}$ (3.74), $306 \mathrm{~nm}$ (3.38); IR (ATR): $v_{\max }$ 3337, 2944, 2831, 1737, 1639, 1547, 1446, 1422, 1329, 1258, 1202, 1157, 1024, 923, $759 \mathrm{~cm}^{-1}$; HRMS $[\mathrm{M}+\mathrm{H}]^{+} m / z=632.3025$ (calcd. for $\mathrm{C}_{29} \mathrm{H}_{42} \mathrm{~N}_{7} \mathrm{O}_{9}{ }^{+}$632.3039), 
$[\mathrm{M}-2 \mathrm{H}+\mathrm{Fe}]^{+} m / z=685.2125$ (calcd. for $\left.\mathrm{C}_{29} \mathrm{H}_{39} \mathrm{~N}_{7} \mathrm{O}_{9}{ }^{+} 685.2142\right) .{ }^{1} \mathrm{H}$ NMR $(500 \mathrm{MHz}$, DMSO- $\left.d_{6}\right) \delta 8.56(\mathrm{~s}, 1 \mathrm{H}), 7.97(\mathrm{~s}, 1 \mathrm{H}), 7.63(\mathrm{~d}, J=7.4 \mathrm{~Hz}, 1 \mathrm{H}), 7.45(\mathrm{t}, J=7.6 \mathrm{~Hz}$, $1 \mathrm{H}), 6.98(\mathrm{~d}, J=7.6 \mathrm{~Hz}, 1 \mathrm{H}), 6.91(\mathrm{t}, J=7.6 \mathrm{~Hz}, 1 \mathrm{H}), 5.00((\mathrm{dd}, J=10.2,8.0 \mathrm{~Hz} 1 \mathrm{H})$, $4.63(\mathrm{dd}, J=10.2,8.1 \mathrm{~Hz}, 1 \mathrm{H}), 4.51(\mathrm{t}, J=8.3 \mathrm{~Hz}, 1 \mathrm{H}), 3.91(\mathrm{dd}, J=6.0,5.4 \mathrm{~Hz}$, $1 \mathrm{H}), 3.75(\mathrm{~d}, J=16.0 \mathrm{~Hz}, 1 \mathrm{H}), 3.66(\mathrm{~d}, J=16.0 \mathrm{~Hz}, 1 \mathrm{H}), 3.53-3.41(\mathrm{~m}, 2 \mathrm{H}), 3.25(\mathrm{dd}$, $J=7.0,5.6 \mathrm{~Hz}, 2 \mathrm{H}), 2.93(\mathrm{t}, J=5.0 \mathrm{~Hz}, 1 \mathrm{H}), 2.55-2.50(\mathrm{~m}, 2 \mathrm{H}), 2.24(\mathrm{~s}, 3 \mathrm{H}), 2.03-$ $1.79(\mathrm{~m}, 6 \mathrm{H}), 1.46-1.36(\mathrm{~m}, 2 \mathrm{H}), 1.29(\mathrm{~s}, 3 \mathrm{H}), 1.11(\mathrm{~s}, 3 \mathrm{H}) .{ }^{13} \mathrm{C}$ NMR $(125 \mathrm{MHz}$, DMSO- $\left.d_{6}\right) \delta 170.6,170.2,169.9,168.4,165.9,163.3,159.1,134.1,128.1,119.0$, $116.7,110.0,77.9,69.4,67.4,62.0,51.7,51.1,47.5,42.2,34.8,32.5,31.7,25.7,25.5$, 25.0, 21.3, 20.9, 19.8 .

\section{Chrome Azurol S (CAS) assay}

Hexadecyltrimethylammonium bromide (CTAB) $(21.9 \mathrm{mg})$ was dissolved in $25 \mathrm{~mL}$ of $\mathrm{H}_{2} \mathrm{O}$ at $35{ }^{\circ} \mathrm{C}$. To this solution were added $1.5 \mathrm{~mL}$ of $1 \mathrm{mM}$ iron(III) chloride solution (prepared by dissolving anhydrous $\mathrm{FeCl} 3$ in a $10 \mathrm{mM}$ aqueous $\mathrm{HCl}$ solution) and $7.5 \mathrm{~mL}$ of a $2 \mathrm{mM}$ aqueous CAS solution at RT. In a separate Erlenmeyer flask, 9.76 $\mathrm{g}$ of 2-(N-morpholino)ethanesulfonic acid (MES) was diluted in $50 \mathrm{~mL}$ of water, and a $50 \% \mathrm{KOH}$ solution was used to adjust the $\mathrm{pH}$ of this solution to 5.6. Then the premade $\mathrm{CTAB}-\mathrm{CAS}-\mathrm{Fe}(\mathrm{III})$ solution was poured into this MES buffer while stirring, and $\mathrm{H}_{2} \mathrm{O}$ was added to make $100 \mathrm{~mL}$ to finish the preparation of the modified CAS assay solution. CTAB-CAS-Fe(III) with MES buffer solution $(100 \mu \mathrm{L})$ was added to each well of the 96-well microplate. Each well was then treated with $100 \mu \mathrm{L}$ of the dilute solution of thalassosamide or deferoxamine mesylate in $\mathrm{H}_{2} \mathrm{O}$ to achieve final concentrations ranging from $1.28 \mathrm{mM}$ to $1.25 \mu \mathrm{M}$. Each reaction was carried out in duplicate. After incubation at $37^{\circ} \mathrm{C}$ for $3 \mathrm{~h}$, the resulting color changes were observed by visual inspection, or the corresponding absorption changes were recorded. Data shown in Figure S16.

\section{Marfey's Analysis}

L-FDLA was synthesized following the experimental procedures in the original report ${ }^{4}$. D-Serine was purchased from TCI America Ltd. L-Serine was purchased from Sigma Aldrich Ltd. Separation of different diastereoisomers was carried out and detected on UHPLC-HRMS system using a linear gradient of $0.3 \mathrm{~mL} / \mathrm{mL}$ of $10 \%-100 \% \mathrm{MeOH} / \mathrm{H}_{2} \mathrm{O}(0.1 \%$ formic acid $)$ over 13 minutes and a hold at $100 \%$ $\mathrm{MeOH}$ for 3 minutes.

Five hundred $\mu \mathrm{g}$ of 1 was dissolved in $400 \mu \mathrm{L}$ of concentrated HI and heated to $110{ }^{\circ} \mathrm{C}$ for 4 hours. The solution was evaporated to dryness and the sample dissolved in $200 \mu \mathrm{L}$ of $1 \mathrm{M} \mathrm{NaHCO}_{3}$. To this was added $170 \mu \mathrm{L}$ of $1 \% \mathrm{~L}-\mathrm{FDLA}$ in acetone and the mixture stirred for 1 hour at $40{ }^{\circ} \mathrm{C}$. The reaction was quenched by the addition of $60 \mu \mathrm{L}$ of $2 \mathrm{M}$ aqueous $\mathrm{HCl}$. The sample was diluted with LC-MS grade $\mathrm{MeOH}$ and analyzed on UHPLC-HRMS.

D-Serine and L-Serine $(0.25 \mathrm{mg})$ were dissolved in $100 \mu \mathrm{L}$ of distilled water and $20 \mu \mathrm{L}$ of $1 \mathrm{M} \mathrm{NaHCO}_{3}$ separately. $100 \mu \mathrm{L}$ of $1 \%$ L-FDLA in acetone was added to both solutions, forming DL and LL pairs. The solutions were heated at $40{ }^{\circ} \mathrm{C}$ for 1 hour, 
diluted with LC-MS grade MeOH and analyzed on UHPLC-HRMS.

Antibacterial Testing

Madurastatin C1 (1), D1 (2) and D2 (3) were tested for antibacterial activity against $M$. luteus (environmental isolate from sponge Ircinia sp.) and Methicillin-resistant Staphylococcus aureus (MRSA) (ATCC \#33591), and MICs were determined using a dilution antimicrobial susceptibility test for aerobic bacteria ${ }^{5}$. Compounds 1, 2 and $\mathbf{3}$ were dissolved in DMSO and serially diluted to 10 concentrations $(0.25-128 \mu \mathrm{g} / \mathrm{mL})$ in 96 -well plates. Vancomycin was used as a positive control and exhibited an MIC of $0.25 \mu \mathrm{g} / \mathrm{mL}$ against $M$. leuteus and MRSA. Madurastatins and vancomycin were tested in triplicates. On each plate, there were six untreated media controls. The plates were incubated at $37{ }^{\circ} \mathrm{C}$ for $18 \mathrm{~h}$. The MIC was determined as the lowest concentration that inhibited visible growth of bacteria.

\section{Whole Genome Sequencing}

DNA extraction of WMMA-1423 was conducted as previously described ${ }^{56}$. The complete genome was sequenced at the University of Wisconsin Biotechnology Center (UWBC) using PacBio RSII (Pacific Biosciences) technology. The assembly of the whole genome was conducted using $\mathrm{Canu}^{7}$. The biosynthetic gene clusters were characterized using antiSMASH $v 4.2^{8}$ and the annotations of the gene clusters were made by blastp against NCBI's non-redundant protein sequences (NR) database.

\section{References}

1. Reasoner, D. J.; Geldreich, E. E. Appl. Environ. Microbiol. 1985, 49, 1-7.

2. Wyche, T. P.; Hou, Y.; Braun, D.; Cohen, H. C.; Xiong, M. P.; Bugni, T. S. J. Org. Chem. 2011, 76, 6542-6547.

3. Alexander, D. B.; Zuberer, D. A. Biol. Fertil. Soils 1991, 12, 39-45.

4. Marfey, P. Carlsberg Res. Commun. 1984, 49, 591-596.

5. National Committee for Clinical Laboratory Standards. Methods for dilution antimicrobial susceptibility tests for bacteria that grow aerobically, 7th ed.; NCCLS: Villanova, PA, USA, 2006; Approved standard M7-A7.

6. Adnani, N.; Chevrette, M. G.; Adibhatla, S. N.; Zhang, F.; Yu, Q.; Braun, D. R.; Nelson, J.; Simpkins, S. W.; McDonald, B. R.; Myers, C. L.; Piotrowski, J. S.; Thompson, C. J.; Currie, C. R.; Li, L.; Rajski, S. R.; Bugni, T. S. ACS Chem. Biol. 2017, 12, 3093-3102.

7. Koren, S.; Walenz, B. P.; Berlin, K.; Miller, J. R.; Bergman, N. H.; Phillippy, A. M. Genome Res. 2017, 27, 722-736.

8. Blin, K.; Wolf, T.; Chevrette, M. G.; Lu, X.; Schwalen, C. J.; Kautsar, S. A.; Duran, H. G. S.; de los Santos, E. L. C.; Kim, H. U.; Nave, M.; Dickschat, J. S.; Mitchell, D. A.; Shelest, E.; Breitling, R.; Takano, E.; Lee, S. Y.; Weber, T.; Medema, M. H. Nucleic Acids Research, 2017, 45, W36-W41. 
Figure S1. ${ }^{1} \mathrm{H}$ NMR spectrum of (-)-madurastatin C1 (1, $\left.400 \mathrm{MHz}, \mathrm{DMSO}-d_{6}\right)$

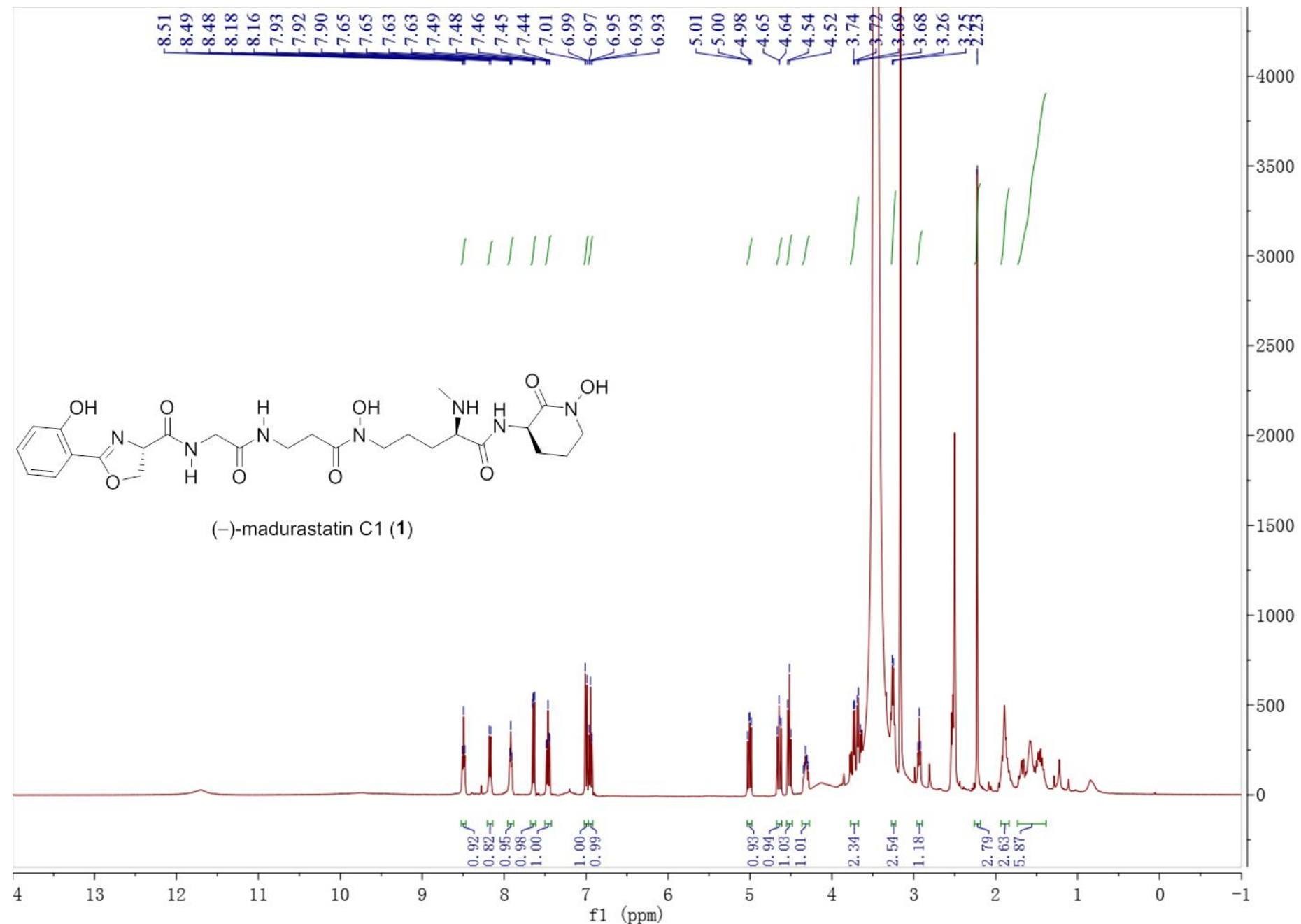


Figure S2. ${ }^{13} \mathrm{C}$ NMR spectrum of (-)-madurastatin C1 $\left(1,101 \mathrm{MHz}, \mathrm{DMSO}-d_{6}\right)$

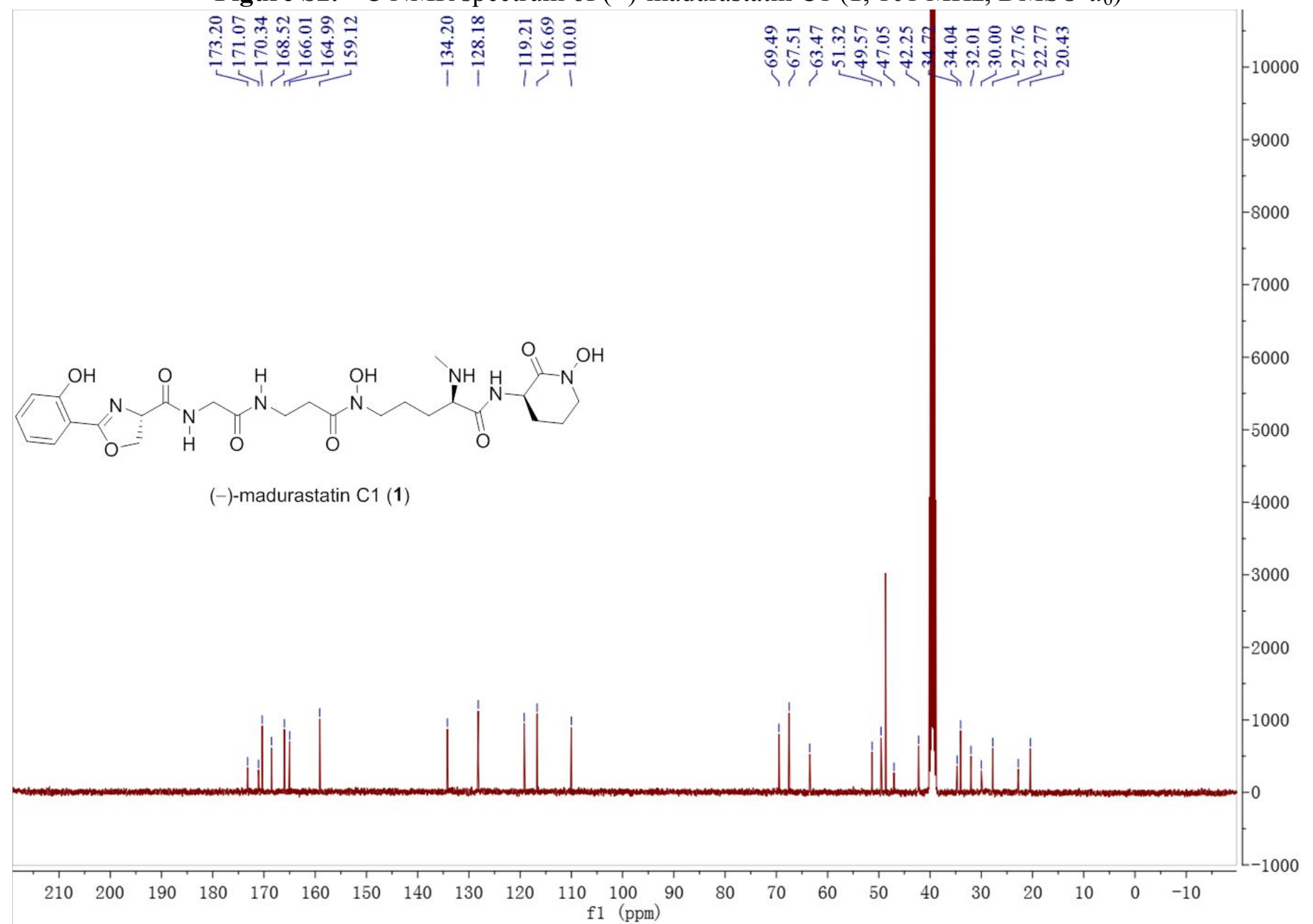


Figure S3. ${ }^{1} \mathrm{H}$ NMR spectrum of madurastatin D1 (2, $\left.500 \mathrm{MHz}, \mathrm{DMSO}-d_{6}\right)$

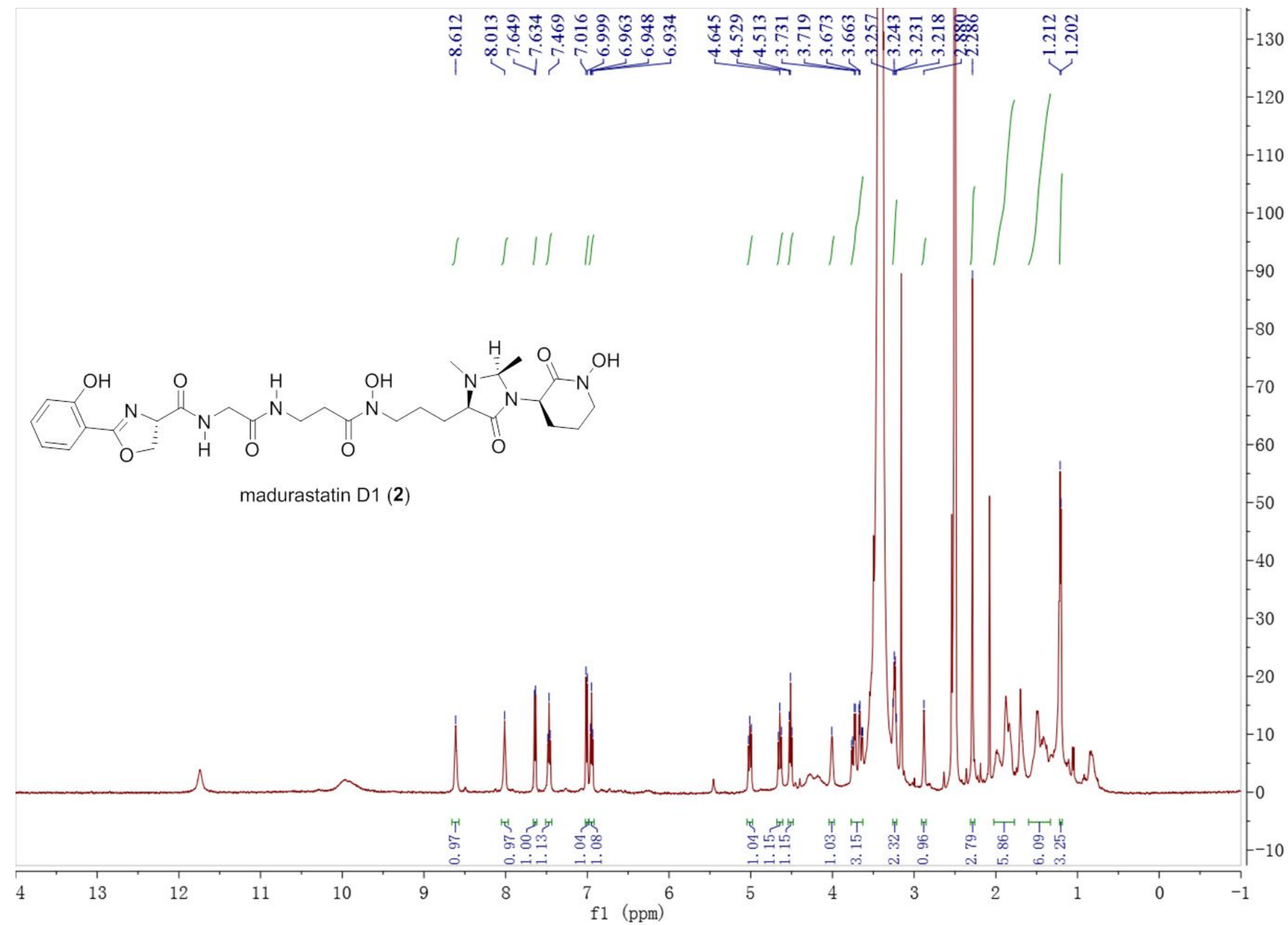


Figure S4. ${ }^{13} \mathrm{C}$ NMR spectrum of madurastatin D1 $\left(2,125 \mathrm{MHz}, \mathrm{DMSO}-d_{6}\right)$

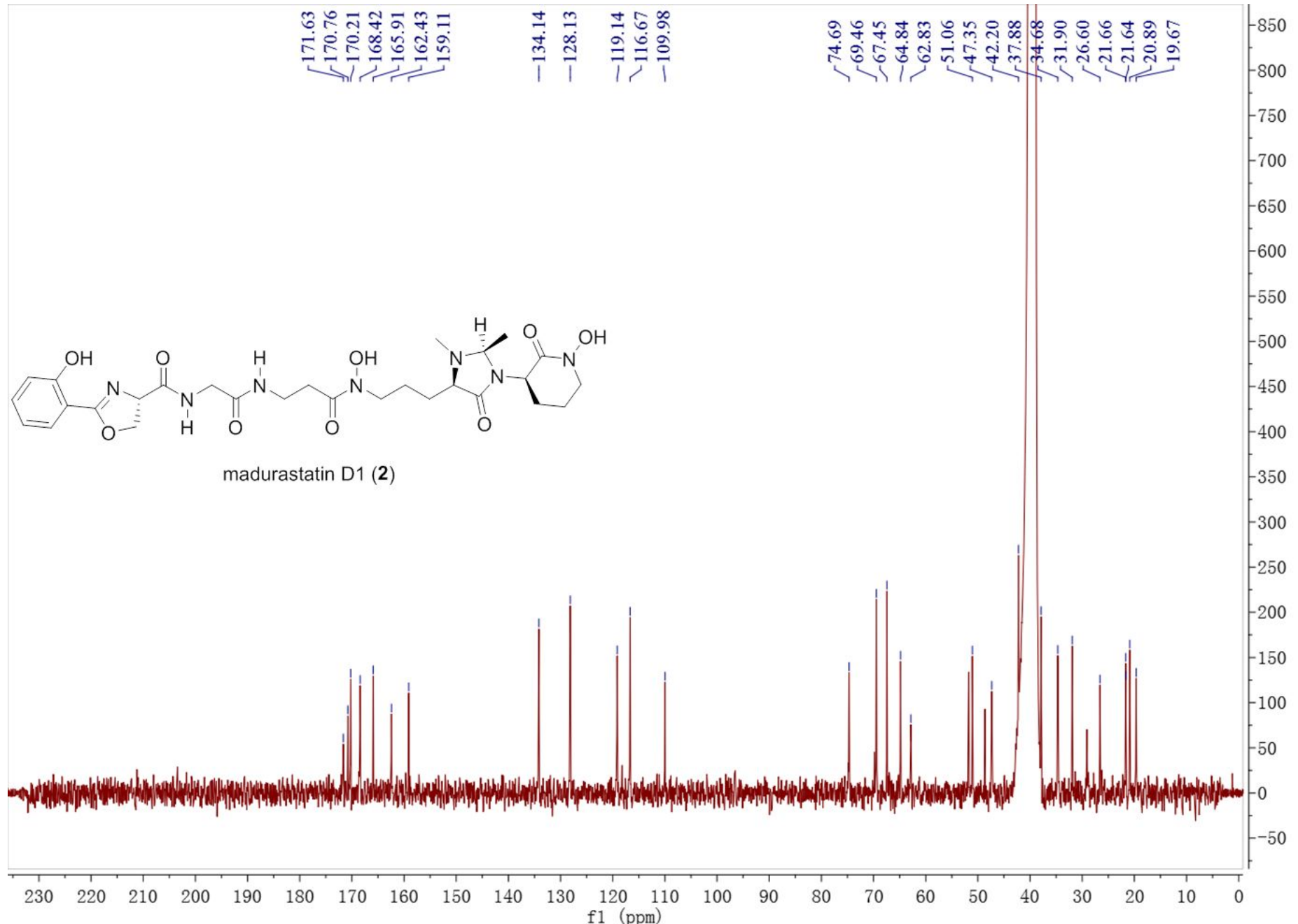


Figure S5. gCOSY spectrum of madurastatin D1 $\left(2,600 \mathrm{MHz}, \mathrm{DMSO}-d_{6}\right)$

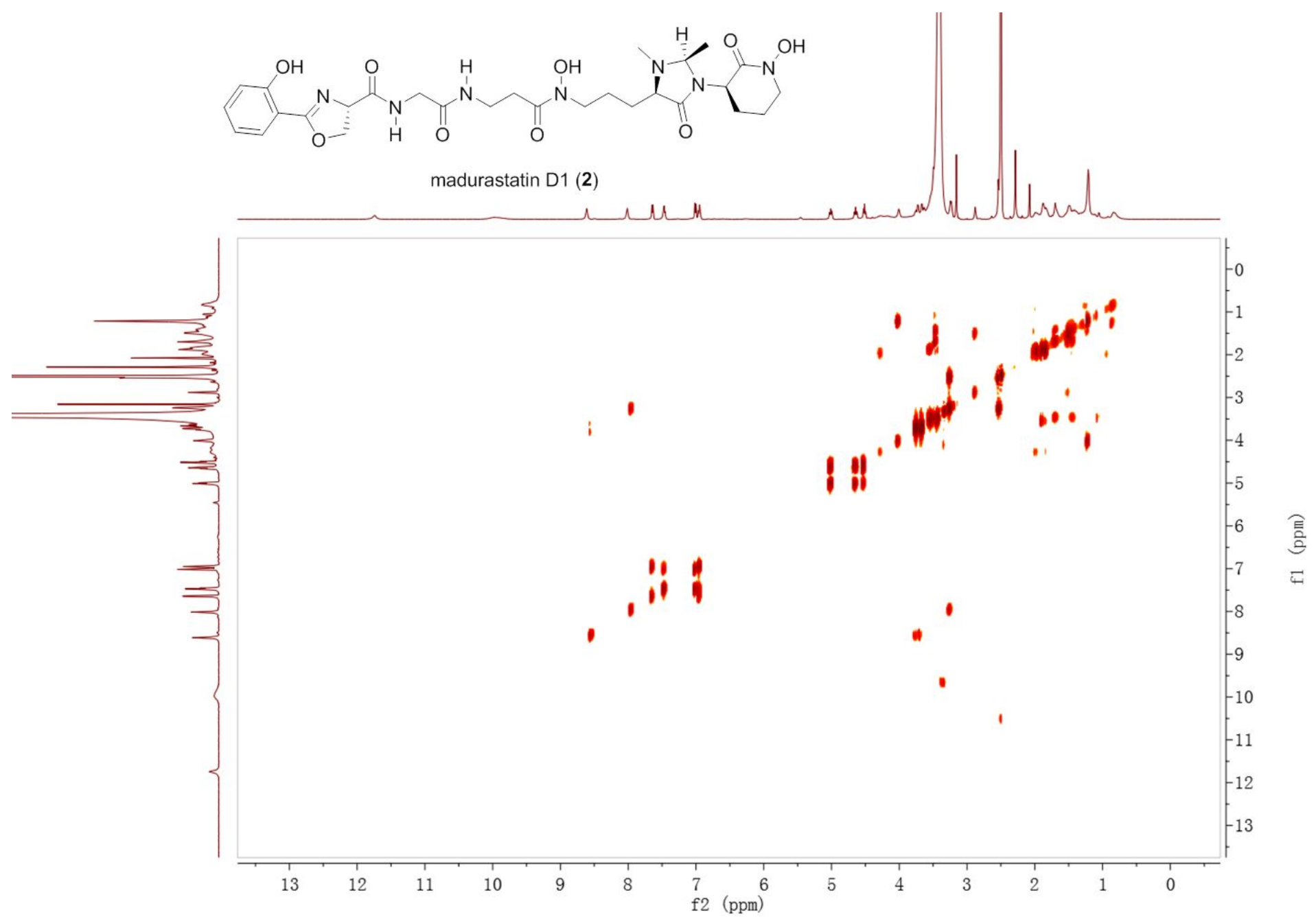


Figure S6. gHSQC spectrum of madurastatin D1 (2, $\left.600 \mathrm{MHz}, \mathrm{DMSO}-d_{6}\right)$

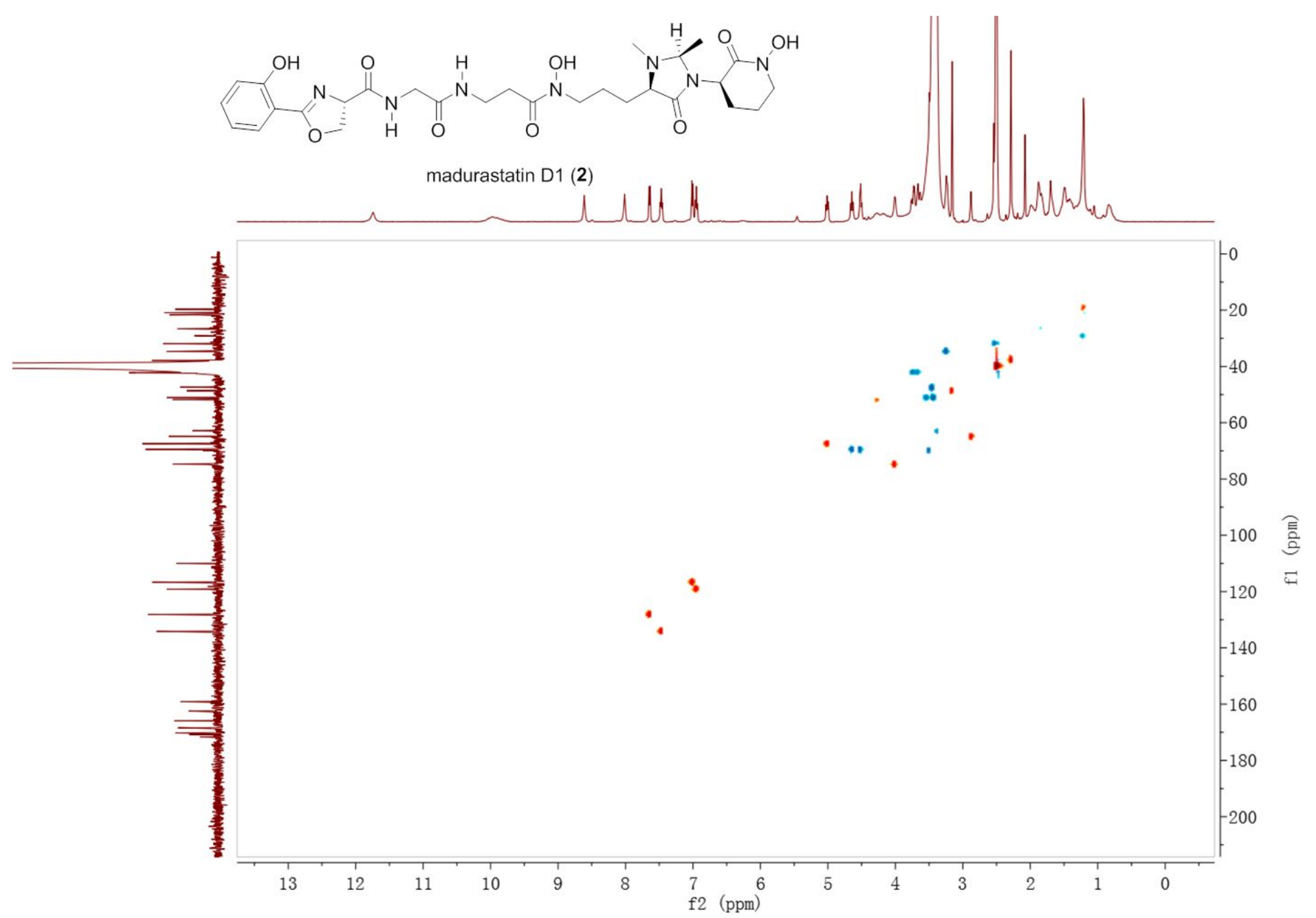


Figure S7. gHMBC spectrum of madurastatin D1 $\left(2,600 \mathrm{MHz}, \mathrm{DMSO}-d_{6}\right)$

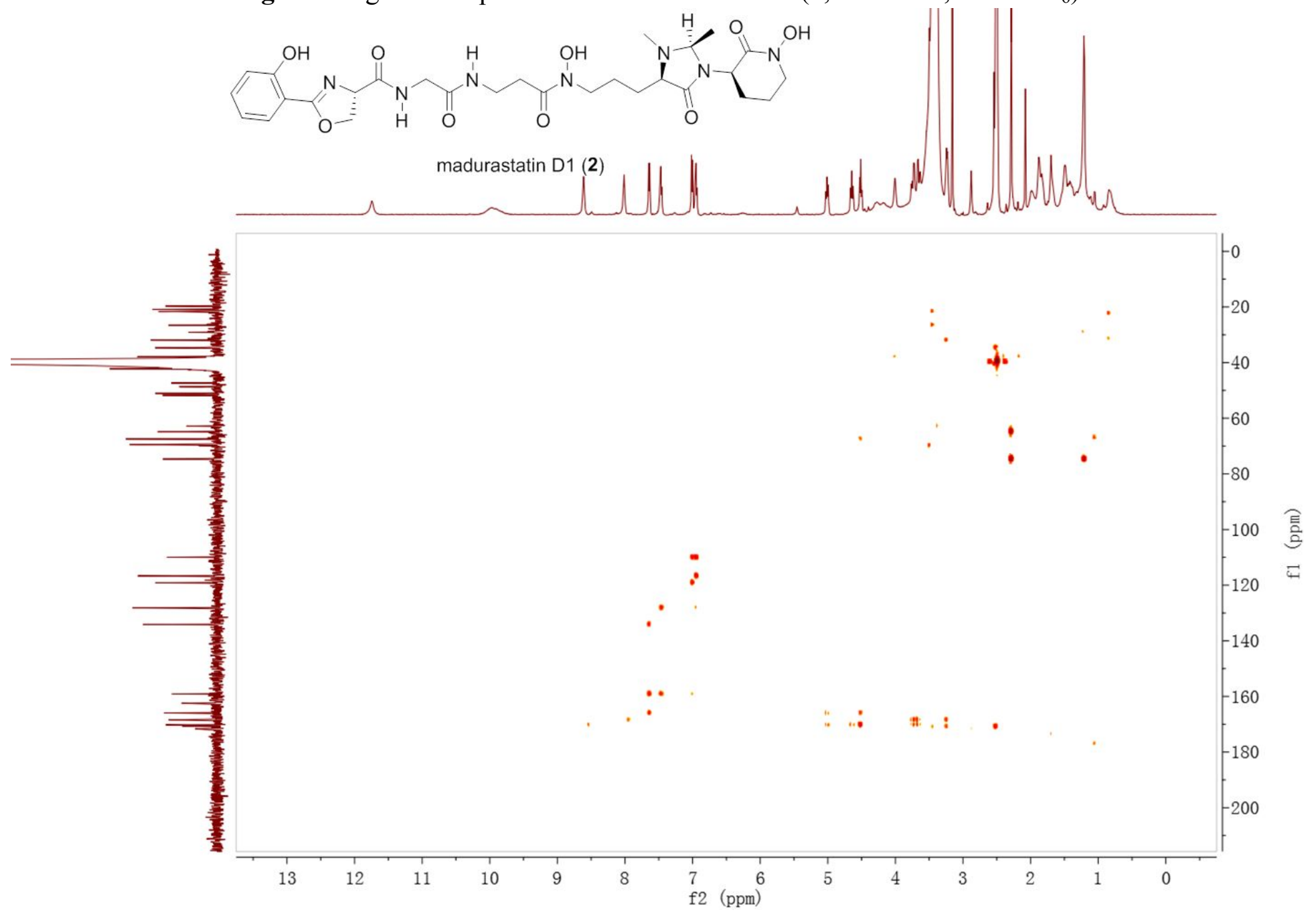


Figure S8. ${ }^{1} \mathrm{H}$ NMR spectrum of madurastatin D2 (3, $\left.500 \mathrm{MHz}, \mathrm{DMSO}-d_{6}\right)$

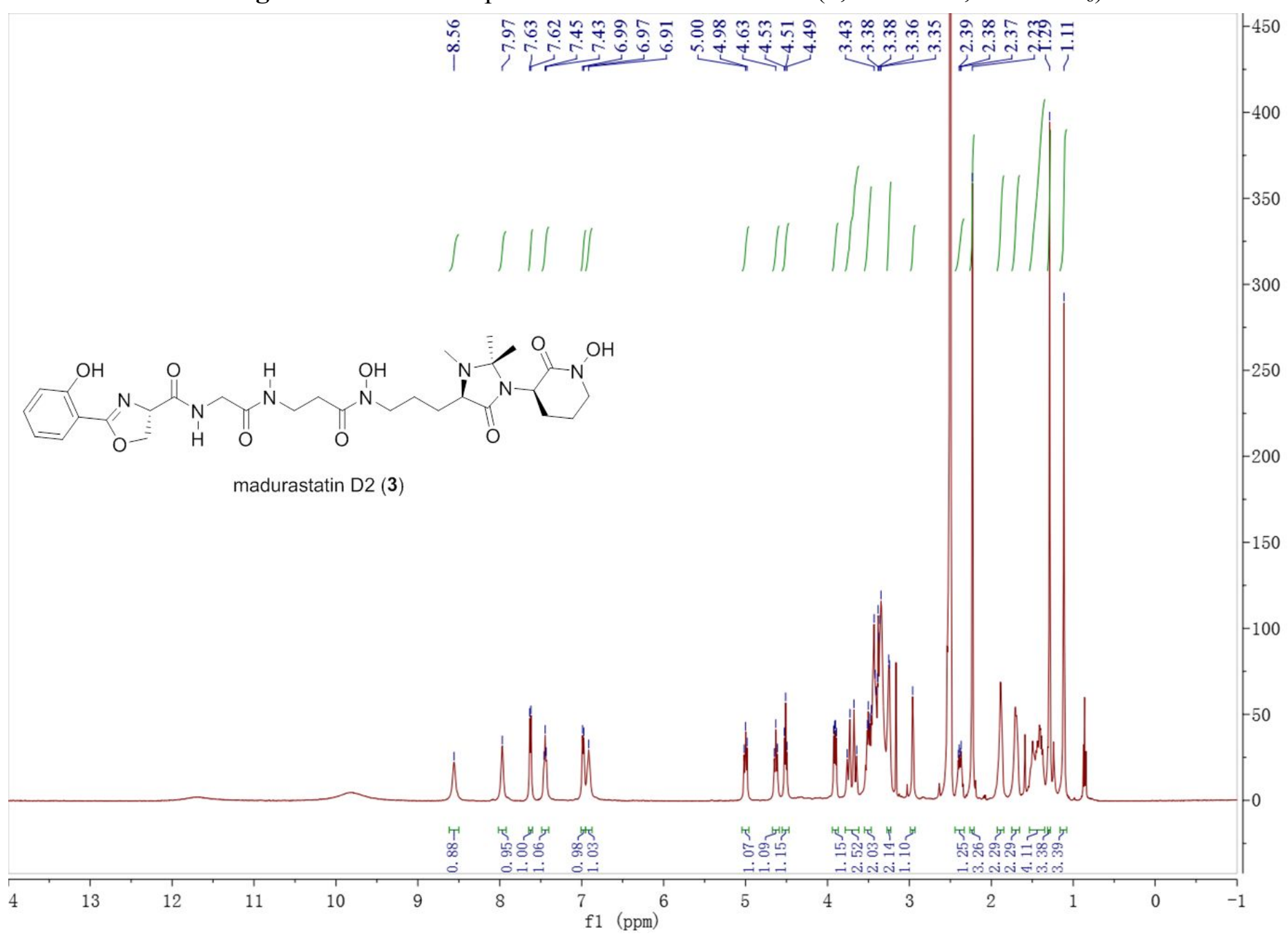


Figure S9. ${ }^{13} \mathrm{C}$ NMR spectrum of madurastatin D2 (3, $\left.125 \mathrm{MHz}, \mathrm{DMSO}-d_{6}\right)$

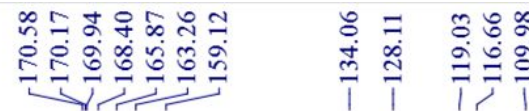

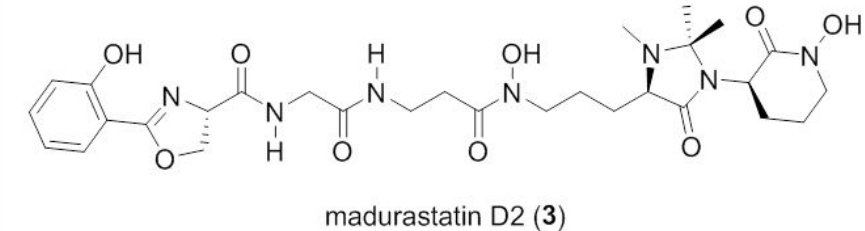

madurastatin D2 (3)

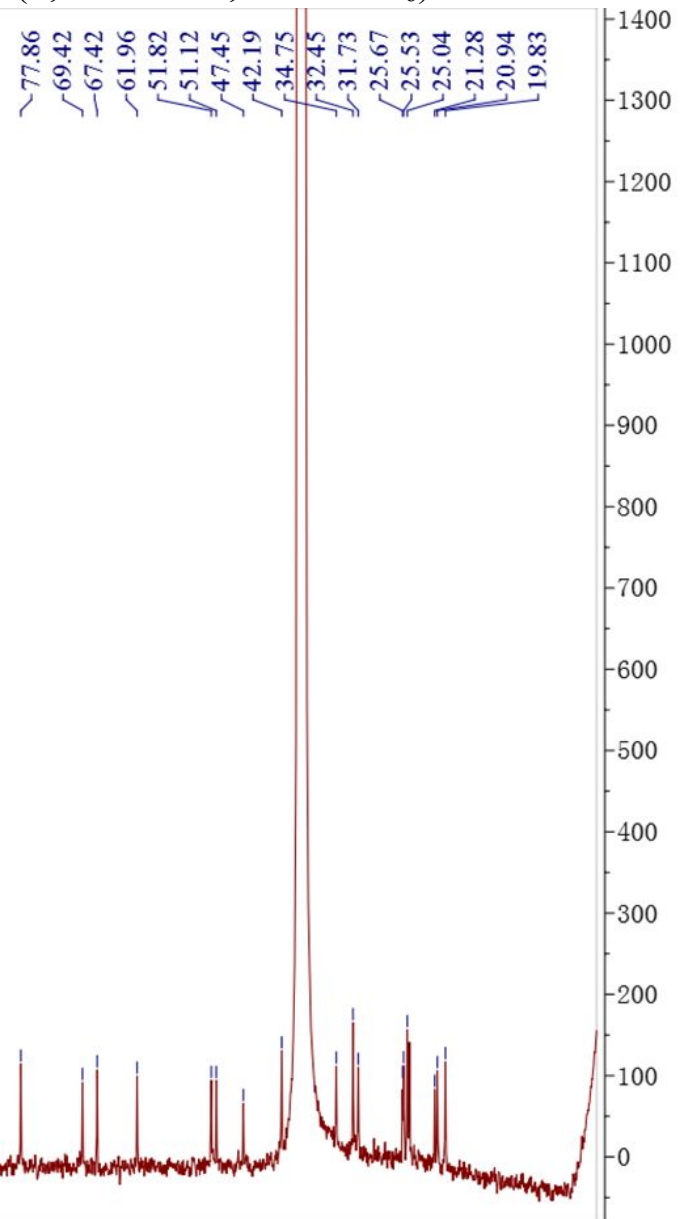

$\begin{array}{lllllll}230 & 220 & 210 & 200 & 190 & 180 & 170\end{array}$ 
Figure S10. gCOSY spectrum of madurastatin D2 $\left(\mathbf{3}, 600 \mathrm{MHz}, \mathrm{DMSO}-d_{6}\right)$
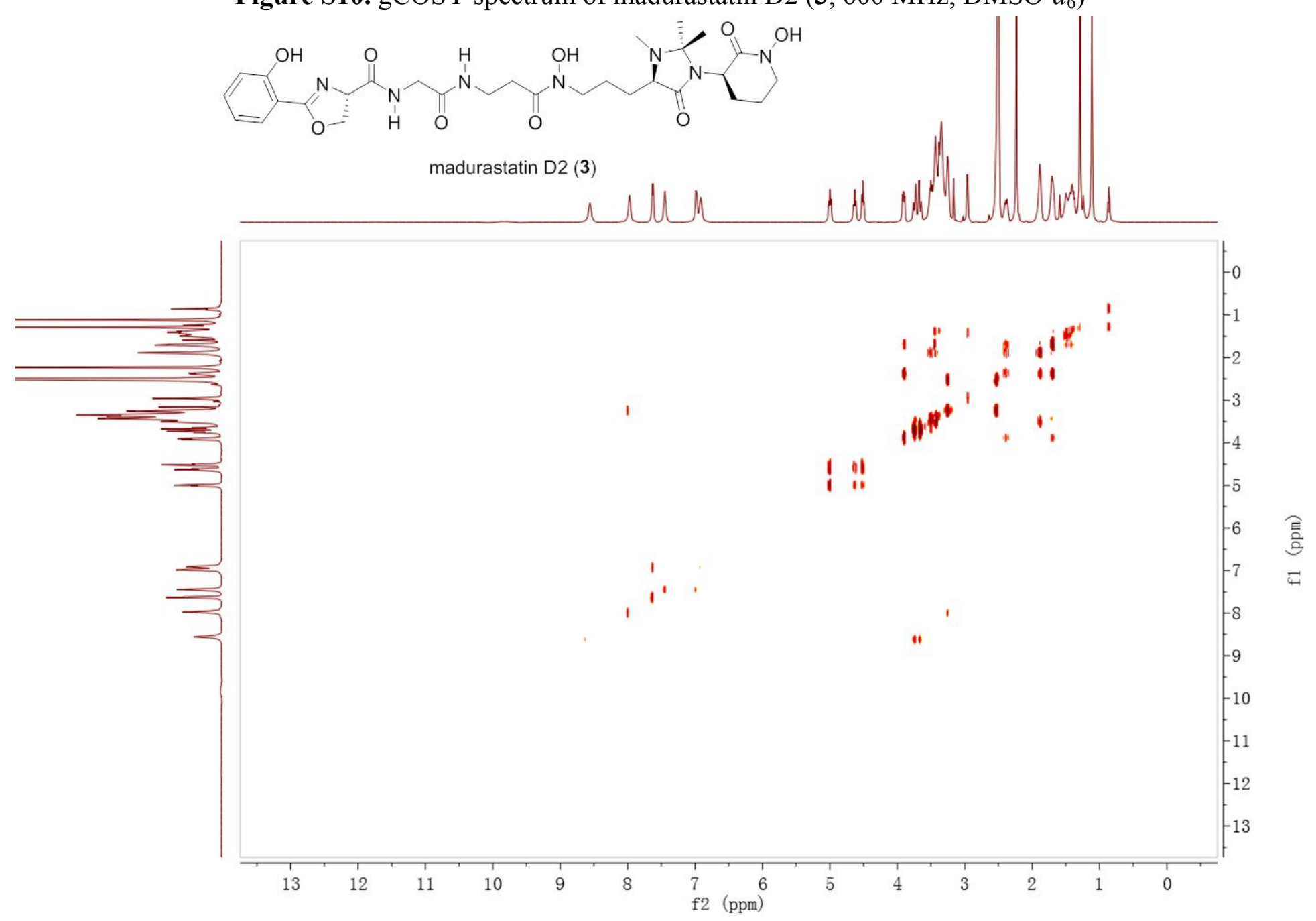
Figure S11. gHSQC spectrum of madurastatin D2 $\left(3,600 \mathrm{MHz}, \mathrm{DMSO}-d_{6}\right)$

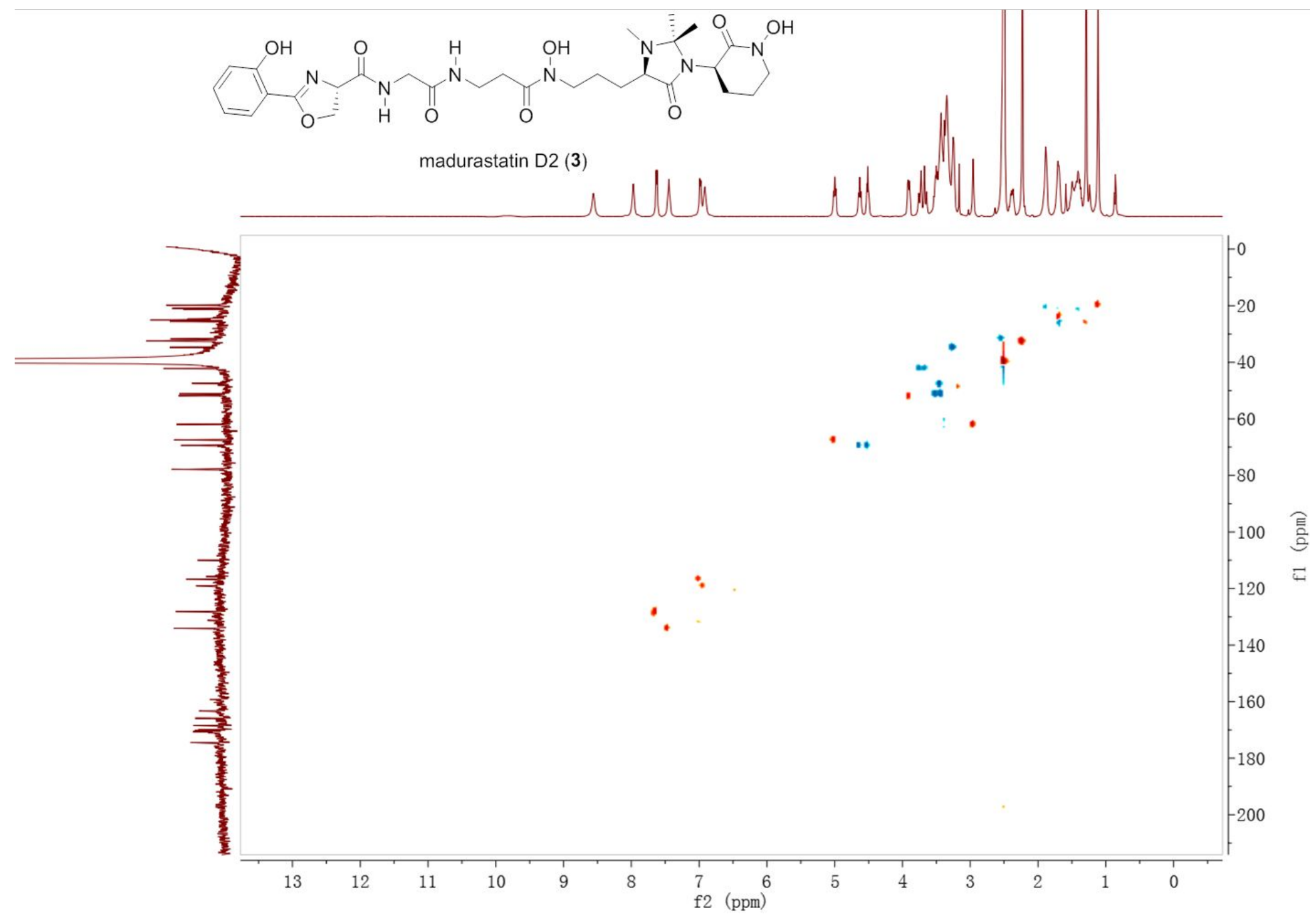


Figure S12. gHMBC spectrum of madurastatin D2 $\left(3,600 \mathrm{MHz}, \mathrm{DMSO}-d_{6}\right)$

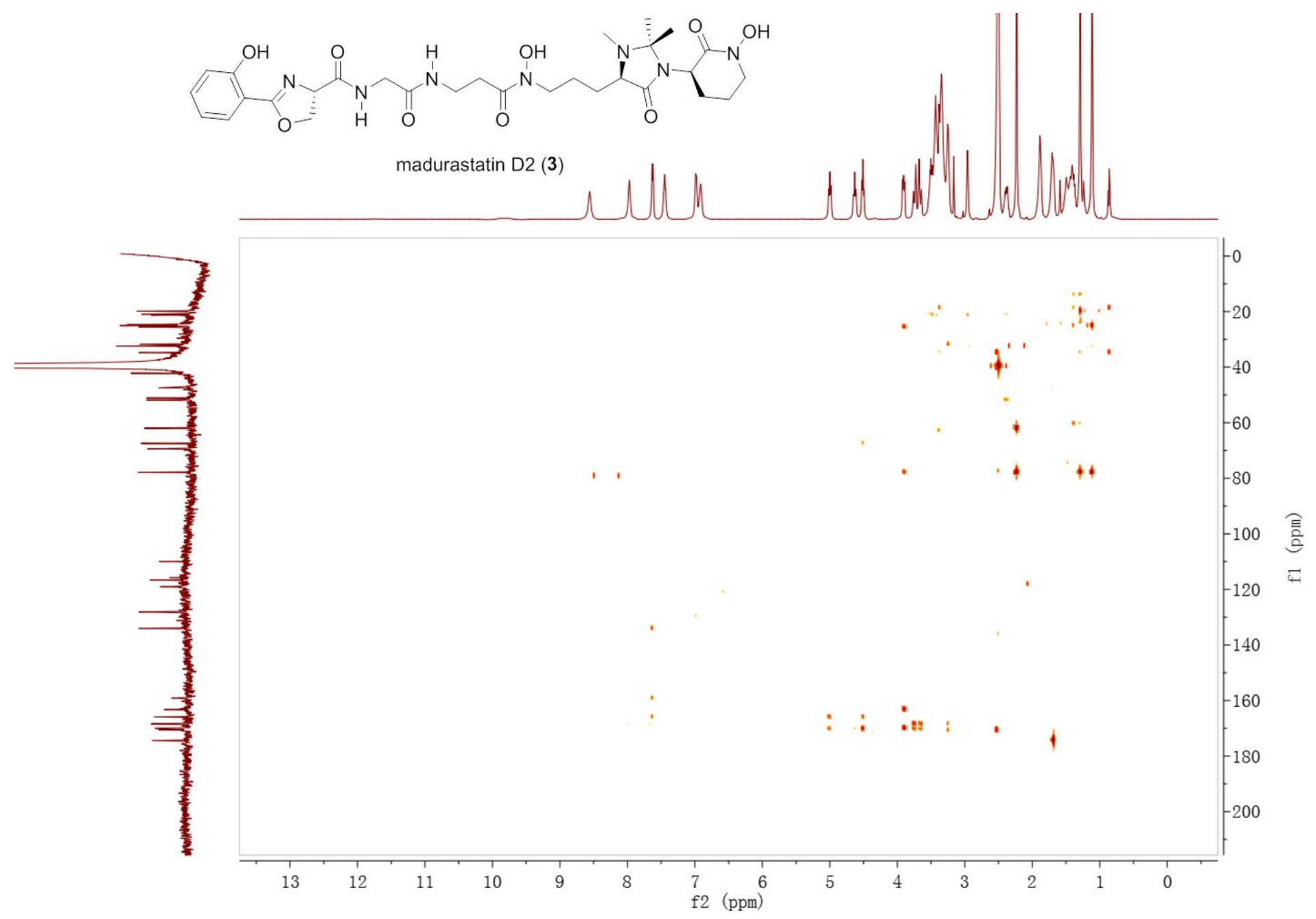


Figure S13. Positive Ion HRESIMS of (-)-madurastatin C1 (1) and the Fe adduct.
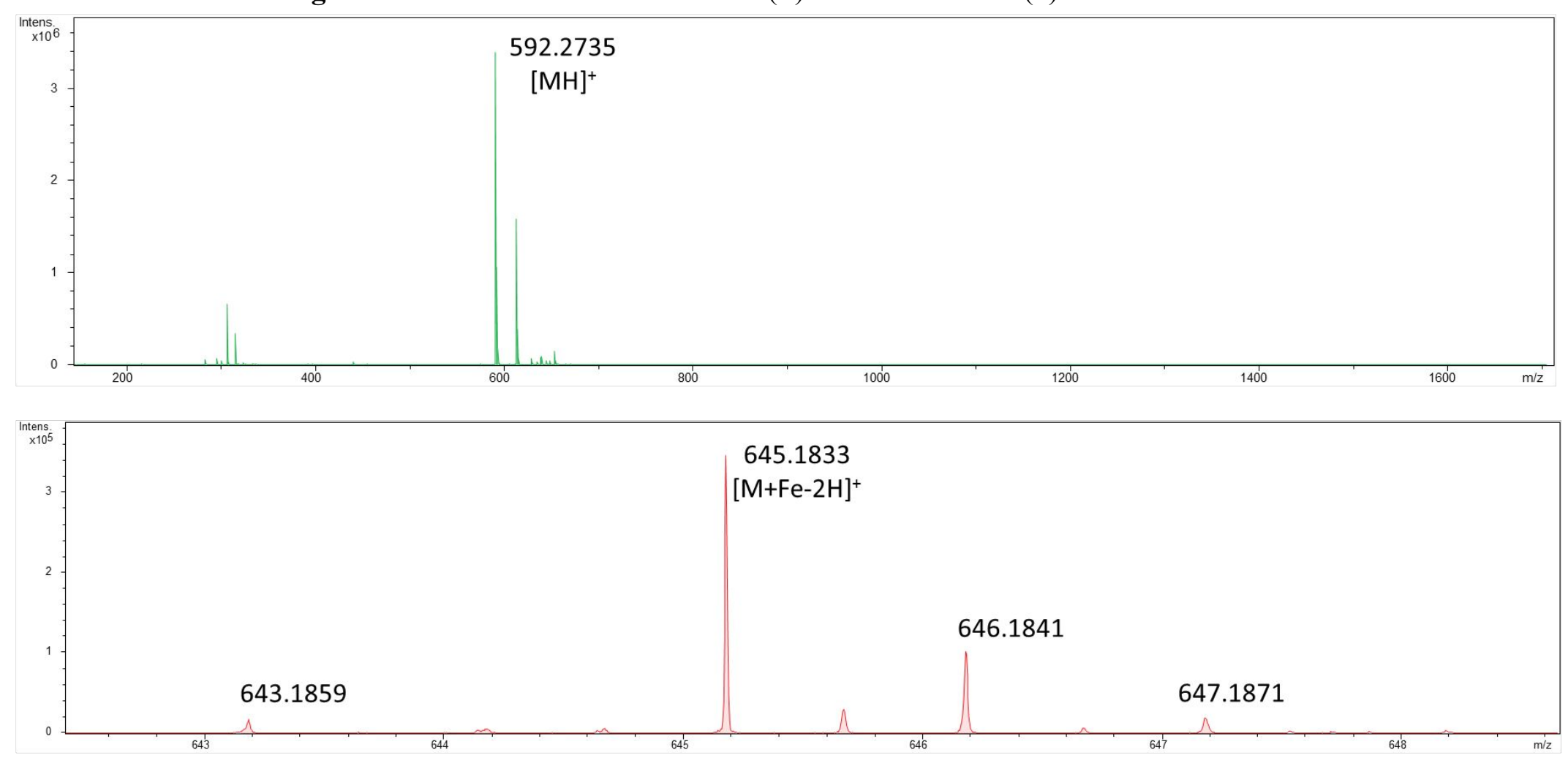
Figure S14. Positive Ion HRESIMS of madurastatin D1 (2) and the Fe adduct.
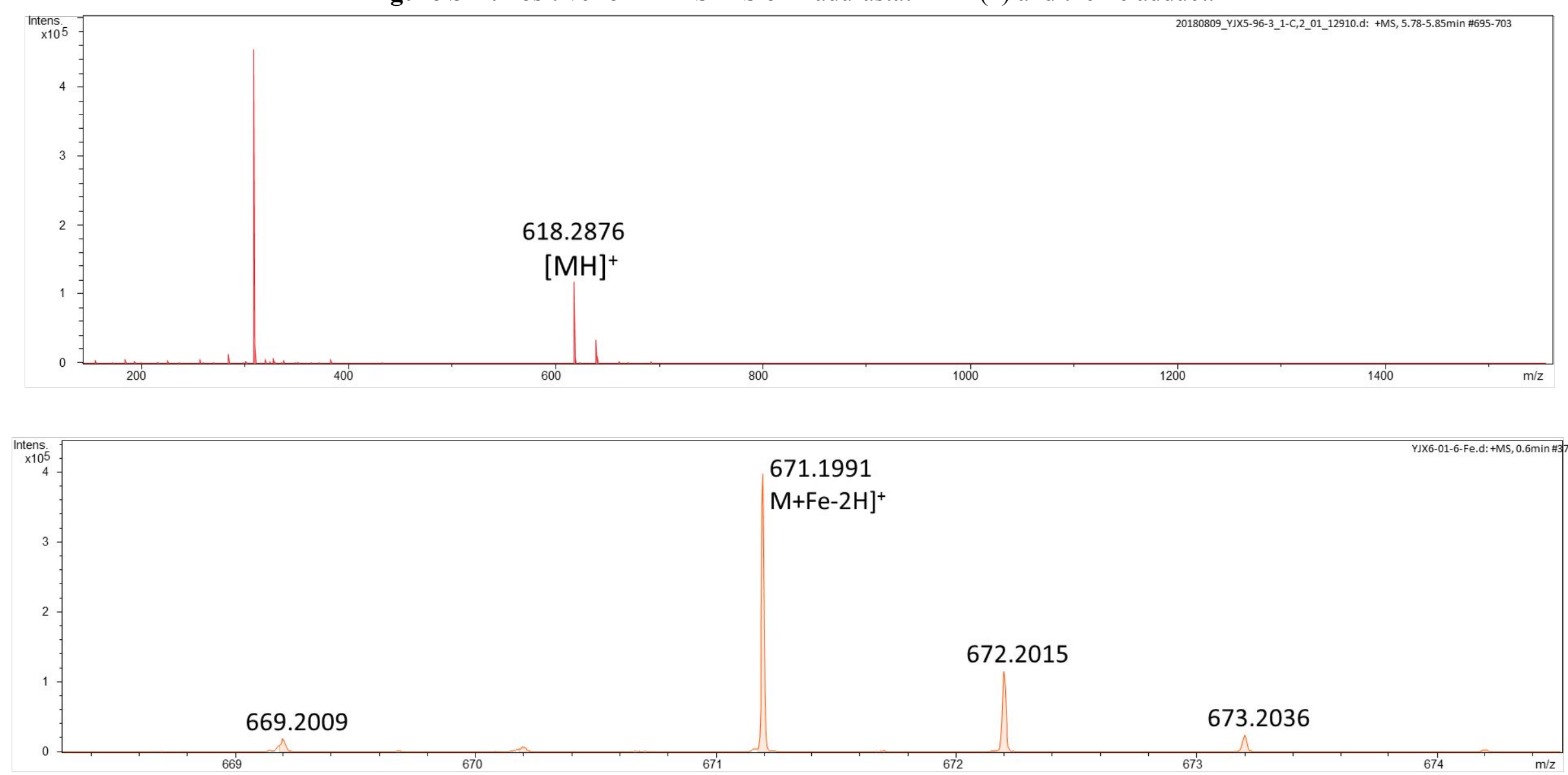
Figure S15. Positive Ion HRESIMS of madurastatin D2 (3) and the Fe adduct.
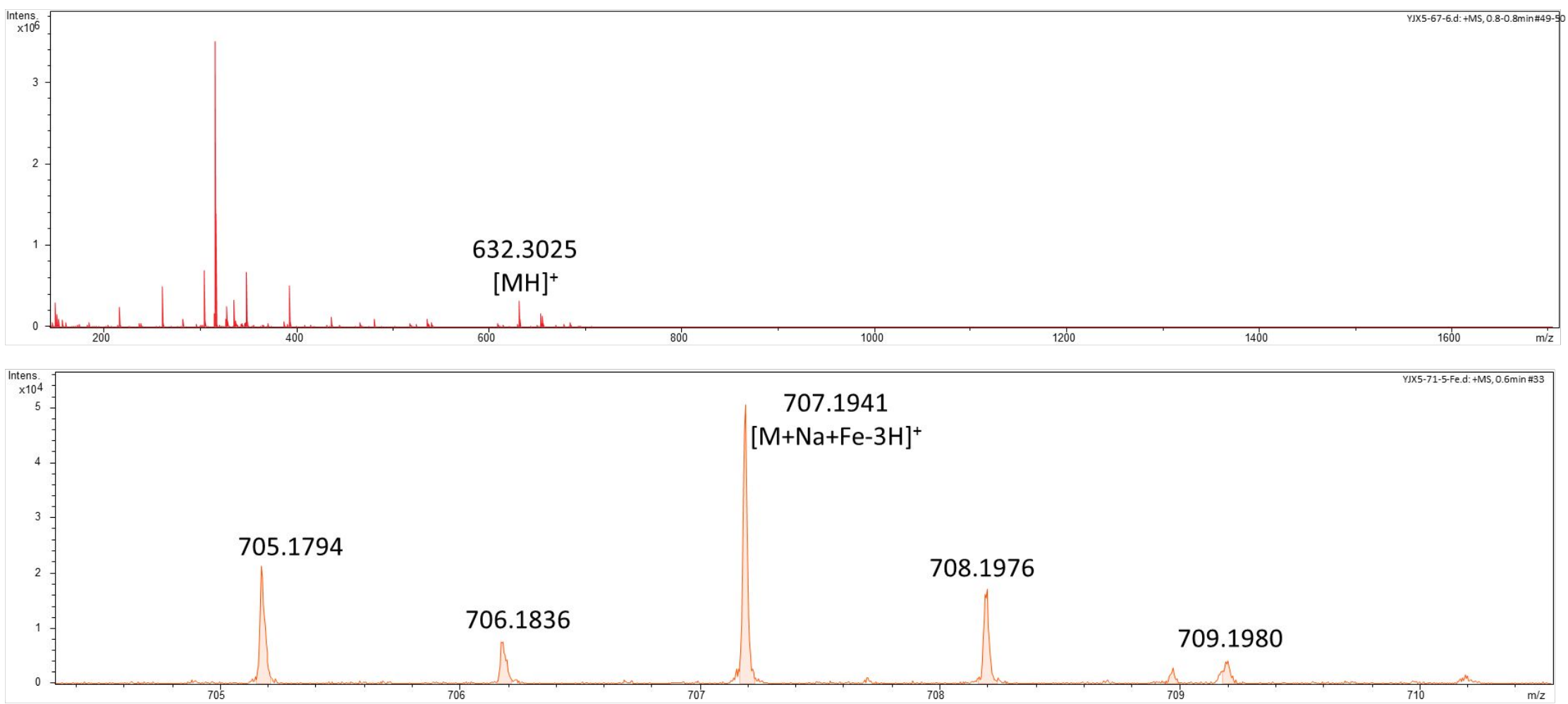
Figure S16. Chrome Azurol S (CAS) assay of compounds 1-3. Each sample run in duplicate.

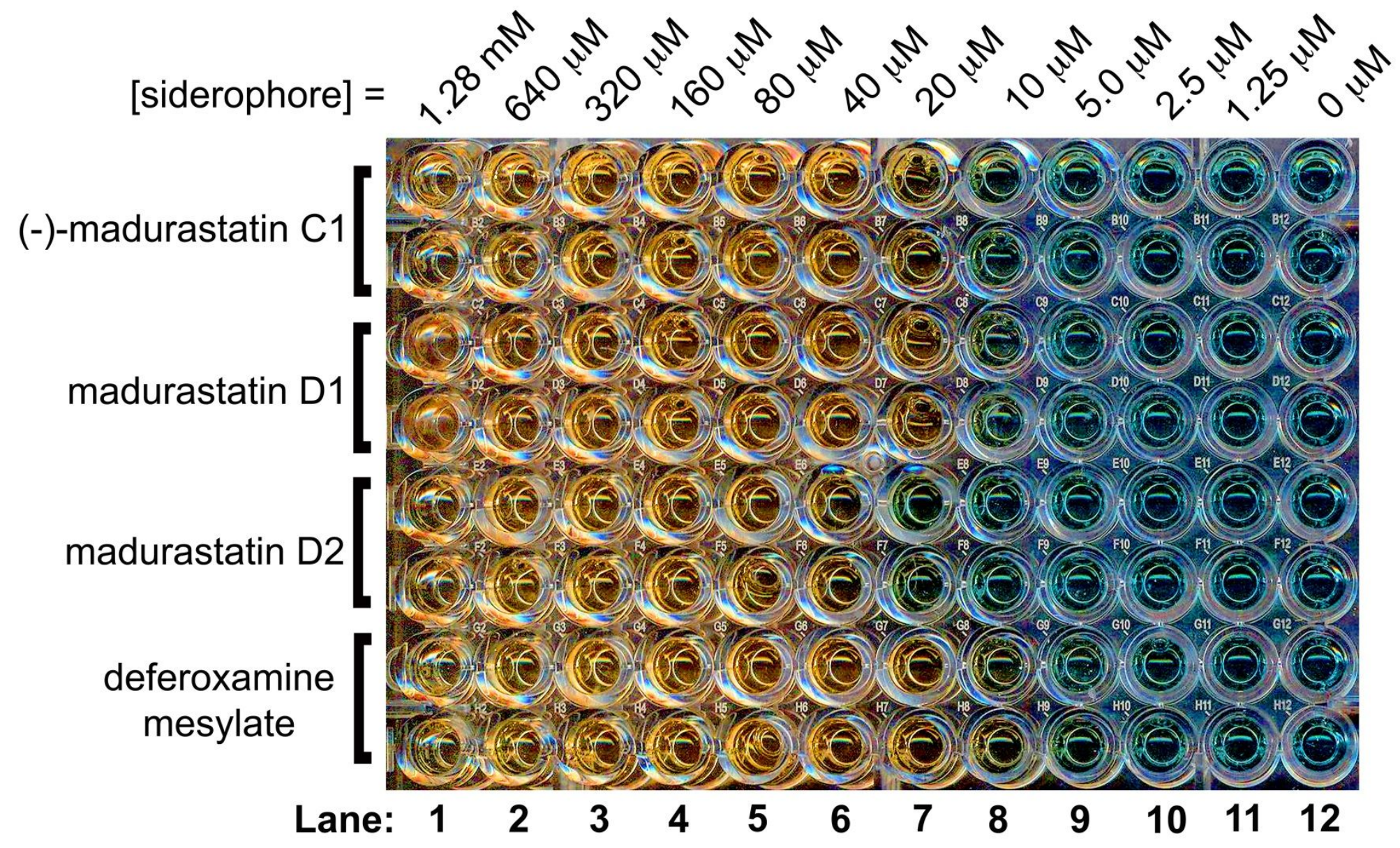


Figure S17. LCMS spectra of Marfey's analysis of (-)-madurastatin C1 (1)

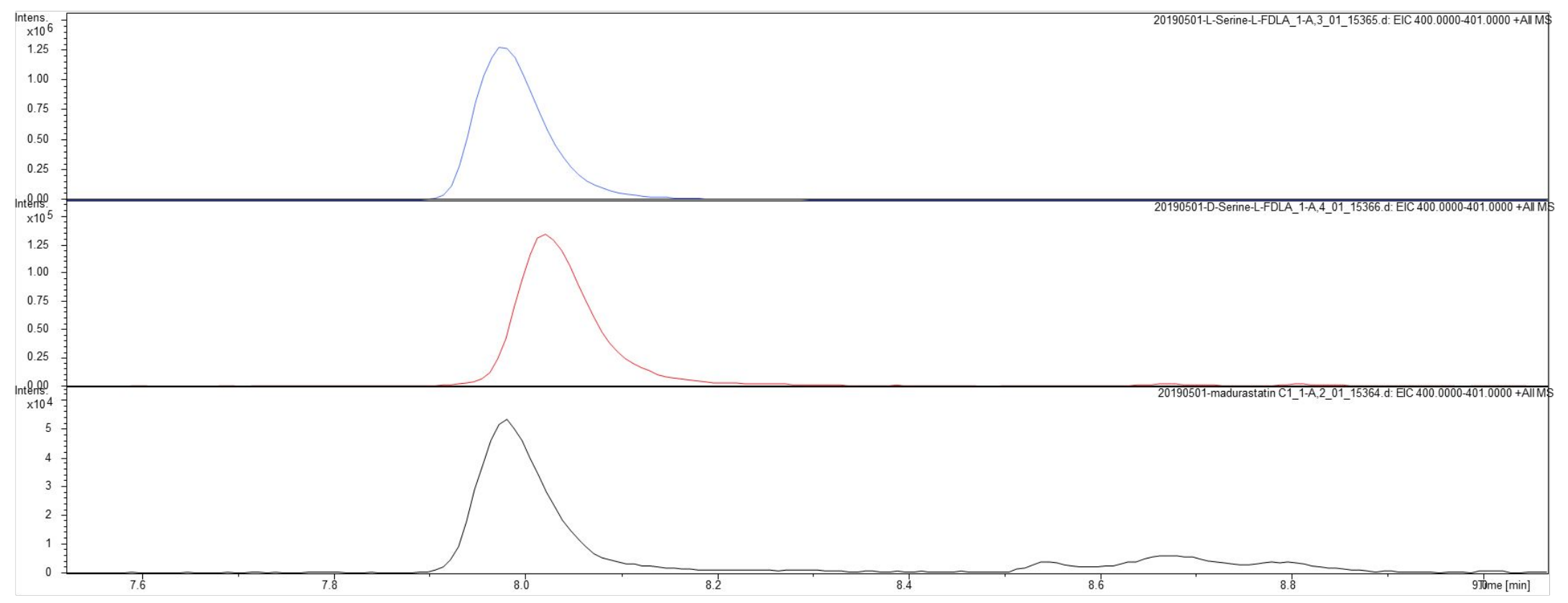

Extracted LC/MS chromatogram spectra of the hydrolyte of L-serine (top trace, blue), D-Serine (middle trace, red) and (-)-Madurastatin C1 (1, bottom trace, black) coupled with L-FDLA. 
Table S1. DFT Calculated ${ }^{13} \mathrm{C}$ NMR chemical shifts of madurastatin D1 (2) diastereoisomers models.

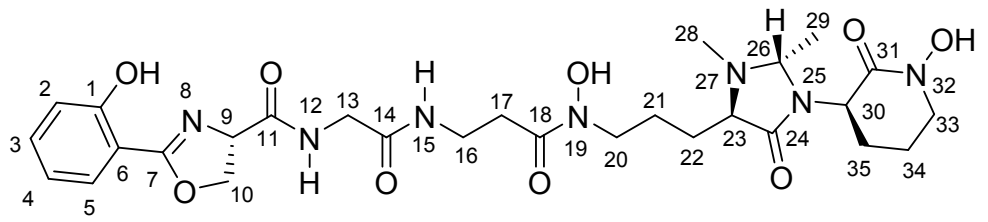

model 1

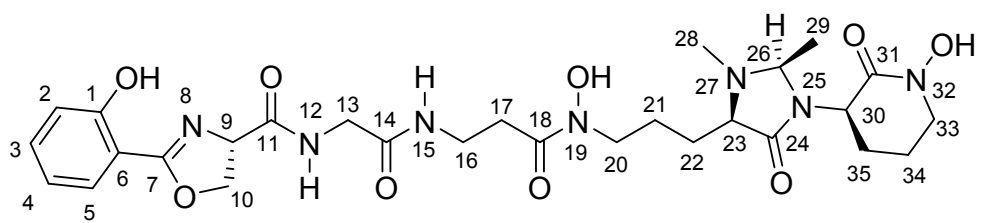

model 2

\begin{tabular}{|c|c|c|c|c|c|}
\hline \multirow{2}{*}{ Position } & \multirow{2}{*}{ Experimental } & \multicolumn{2}{|c|}{ Model 1} & \multicolumn{2}{|c|}{ Model 2} \\
\hline & & Calcd. (ppm) & $\delta(\mathrm{ppm})$ & Calcd. (ppm) & $\delta(\mathrm{ppm})$ \\
\hline 1 & 159.1 & 161.0 & -1.9 & 160.6 & -1.5 \\
\hline 2 & 116.7 & 117.2 & -0.5 & 117.7 & -1.0 \\
\hline 3 & 134.1 & 133.0 & 1.1 & 134.1 & 0.0 \\
\hline 4 & 119.1 & 118.4 & 0.7 & 119.5 & -0.4 \\
\hline 5 & 128.1 & 130.7 & -2.6 & 130.7 & -2.6 \\
\hline 6 & 110 & 114.2 & -4.2 & 113.9 & -3.9 \\
\hline 7 & 165.9 & 160.0 & 5.9 & 162.0 & 3.9 \\
\hline 9 & 67.5 & 68.7 & -1.2 & 69.5 & -2.0 \\
\hline 10 & 69.5 & 71.3 & -1.8 & 70.1 & -0.6 \\
\hline 11 & 170.2 & 168.1 & 2.1 & 172.3 & -2.1 \\
\hline 13 & 42.2 & 43.1 & -0.9 & 47.9 & -5.7 \\
\hline 14 & 168.4 & 172.4 & -4.0 & 168.2 & 0.2 \\
\hline 16 & 34.7 & 39.3 & -4.6 & 41.2 & -6.5 \\
\hline 17 & 31.9 & 33.9 & -2.0 & 37.0 & -5.1 \\
\hline 18 & 170.8 & 171.6 & -0.8 & 172.1 & -1.3 \\
\hline 20 & 47.4 & 46.1 & 1.3 & 48.8 & -1.4 \\
\hline 21 & 21.6 & 25.8 & -4.2 & 25.5 & -3.9 \\
\hline 22 & 26.6 & 26.2 & 0.4 & 31.0 & -4.4 \\
\hline 23 & 64.8 & 65.3 & -0.5 & 67.2 & -2.4 \\
\hline 24 & 171.6 & 176.7 & -5.1 & 173.8 & -2.2 \\
\hline 26 & 74.7 & 78.9 & -4.2 & 79.4 & -4.7 \\
\hline 28 & 37.9 & 35.5 & 2.4 & 39.9 & -2.0 \\
\hline 29 & 19.7 & 17.3 & 2.4 & 21.4 & -1.7 \\
\hline 30 & 51.8 & 58.9 & -7.1 & 57.4 & -5.6 \\
\hline 31 & 162.4 & 165.3 & -2.9 & 164.3 & -1.9 \\
\hline 33 & 51.1 & 52.8 & -1.7 & 51.5 & -0.4 \\
\hline 34 & 21.6 & 25.1 & -3.5 & 25.0 & -3.4 \\
\hline 35 & 20.9 & 26.6 & -5.7 & 27.1 & -6.2 \\
\hline
\end{tabular}


Table S2. DP4 probability calculation of madurastatin D1 (2) diastereoisomer models

\begin{tabular}{ccc}
\hline Models & Configuration & DP4 Probability \\
\hline 1 & $9 S, 23 R, 26 S, 30 R$ & $0 \%$ \\
2 & $9 S, 23 R, 26 R, 30 R$ & $100 \%$ \\
\hline
\end{tabular}


Table S3. Annotations for the mad biosynthetic gene cluster

\begin{tabular}{|c|c|c|c|c|c|}
\hline Locus & Gene & Proposed Annotation & Notable Homologs & Protein \% ID & Organism \\
\hline Cluster 1 & Mad1 & acyl-CoA dehydrogenase & WP_089316159.1 & 96 & Actinomadura mexicana \\
\hline Cluster 1 & Mad2 & hypothetical protein & WP_067467552.1 & 90 & Actinomadura macra \\
\hline Cluster 1 & Mad3 & hypothetical protein & WP_021600299.1 & 95 & Actinomadura madurae \\
\hline Cluster 1 & Mad4 & methyltransferase domain-containing protein & WP_130186440.1 & 64 & Massilia lutea \\
\hline Cluster 1 & Mad5 & YbdK family carboxylate-amine ligase & WP_089316162.1 & 92 & Actinomadura mexicana \\
\hline Cluster 1 & Mad6 & sensor histidine kinase & WP_089316163.1 & 82 & Actinomadura mexicana \\
\hline Cluster 1 & Mad7 & aminoglycoside phosphotransferase & WP_132196454.1 & 81 & $\begin{array}{l}\text { Actinomadura } \\
\text { darangshiensis }\end{array}$ \\
\hline Cluster 1 & Mad 8 & DUF2269 domain-containing protein & WP_131742939.1 & 73 & $\begin{array}{l}\text { Actinomadura sp. LMG } \\
30035\end{array}$ \\
\hline Cluster 1 & Mad 9 & hypothetical DoxX membrane protein & WP_089316164.1 & 86 & Actinomadura mexicana \\
\hline Cluster 1 & Mad 10 & GNAT family $\mathrm{N}$-acetyltransferase & WP_089316165.1 & 92 & Actinomadura mexicana \\
\hline Cluster 1 & Mad 11 & SAM-dependent methyltransferase & WP_132196462.1 & 80 & $\begin{array}{l}\text { Actinomadura } \\
\text { darangshiensis }\end{array}$ \\
\hline Cluster 1 & Mad 12 & acyltransferase & WP_089316167.1 & 93 & Actinomadura mexicana \\
\hline Cluster 1 & Mad 13 & PucR family transcriptional regulator & WP_033331132.1 & 94.06 & Actinomadura madurae \\
\hline Cluster 1 & Mad 14 & esterase & WP_132205301.1 & 78.78 & $\begin{array}{l}\text { Actinomadura } \\
\text { darangshiensis }\end{array}$ \\
\hline Cluster 1 & Mad 15 & hypothetical protein & WP_132205297.1 & 91.35 & $\begin{array}{l}\text { Actinomadura } \\
\text { darangshiensis }\end{array}$ \\
\hline Cluster 1 & Mad 16 & 1-phosphofructokinase & WP_131941588.1 & 92.42 & $\begin{array}{l}\text { Actinomadura } \\
\text { bangladeshensis }\end{array}$ \\
\hline Cluster 1 & Mad 17 & DeoR/GlpR transcriptional regulator & WP_132203352.1 & 94.85 & $\begin{array}{l}\text { Actinomadura } \\
\text { darangshiensis }\end{array}$ \\
\hline Cluster 1 & Mad 18 & $\mathrm{ABC}$ transporter substrate-binding protein & WP_089316172.1 & 96.46 & Actinomadura mexicana \\
\hline Cluster 1 & Mad 19 & $\begin{array}{l}\text { branched-chain amino acid } \mathrm{ABC} \text { transporter } \\
\text { permease }\end{array}$ & WP_075021706.1 & 89.06 & Actinomadura madurae \\
\hline Cluster 1 & Mad 20 & $\begin{array}{l}\text { branched-chain amino acid ABC transporter } \\
\text { permease }\end{array}$ & WP_089316174.1 & 96.58 & Actinomadura mexicana \\
\hline Cluster 1 & Mad 21 & $\mathrm{ABC}$ transporter ATP-binding protein & WP_089328063.1 & 90.21 & Actinomadura meyerae \\
\hline Cluster 1 & Mad 22 & $\mathrm{ABC}$ transporter ATP-binding protein & WP_089316176.1 & 92.66 & Actinomadura mexicana \\
\hline Cluster 1 & Mad 23 & TetR/AcrR family transcriptional regulator & WP_089316177.1 & 95.50 & Actinomadura mexicana \\
\hline Cluster 1 & Mad 24 & long-chain fatty acid--CoA ligase & WP_089316224.1 & 93.05 & Actinomadura mexicana \\
\hline Cluster 1 & Mad 25 & MFS transporter & WP_089328068.1 & 72.63 & Actinomadura meyerae \\
\hline Cluster 1 & Mad 26 & hypothetical esterase & WP_089316178.1 & 92.66 & Actinomadura mexicana \\
\hline Cluster 1 & Mad 27 & MbtH family protein & CahE & 63 & Streptomyces gandocaensis \\
\hline Cluster 1 & $\operatorname{Mad} 28$ & $\begin{array}{l}\text { lysine } \mathrm{N}(6) \text {-hydroxylase/L-ornithine } \\
\mathrm{N}(5) \text {-oxygenase family protein }\end{array}$ & cahMO & 57 & Streptomyces gandocaensis \\
\hline Cluster 1 & Mad 29 & $\begin{array}{l}\text { iron-siderophore } \mathrm{ABC} \text { transporter } \\
\text { substrate-binding protein }\end{array}$ & WP_089316225.1 & 89.85 & Actinomadura mexicana \\
\hline Cluster 1 & Mad 30 & non-ribosomal peptide synthetase & AMYAL_RS0130210 & 47 & Amycolatopsis alba \\
\hline Cluster 1 & Mad 31 & salicylate synthase & AmcL & 59 & Amycolatopsis sp. AA4 \\
\hline Cluster 1 & Mad 32 & Fpg/Nei family DNA glycosylase & WP_089328073.1 & 73.51 & Actinomadura meyerae \\
\hline Cluster 1 & Mad 33 & hypothetical protein & WP_089316188.1 & 60.54 & Actinomadura mexicana \\
\hline Cluster 1 & Mad 34 & hypothetical protein & WP_117402065.1 & 70.83 & $\begin{array}{l}\text { Actinomadura sp. } \\
\text { LHW52907 }\end{array}$ \\
\hline Cluster 1 & Mad 35 & exonuclease & WP_117402874.1 & 75.86 & $\begin{array}{l}\text { Actinomadura sp. } \\
\text { LHW52907 }\end{array}$ \\
\hline Cluster 1 & Mad 36 & cysteine hydrolase & WP_084521215.1 & 96.67 & Nocardia uniformis \\
\hline Cluster 1 & Mad 37 & hypothetical protein & WP_112468680.1 & 87.66 & Streptomyces triticisoli \\
\hline Cluster 1 & Mad 38 & alpha-ketoglutarate-dependent dioxygenase AlkB & WP_075024900.1 & 83.90 & Actinomadura madurae \\
\hline
\end{tabular}




\begin{tabular}{|c|c|c|c|c|c|}
\hline Cluster 1 & Mad 39 & hypothetical protein & WP_131732831.1 & 63.64 & Actinomadura formosensis \\
\hline Cluster 1 & $\operatorname{Mad} 40$ & TROVE domain-containing protein & WP_131888826.1 & 82.28 & Actinomadura sp. $\mathrm{H} 3 \mathrm{C} 3$ \\
\hline Cluster 1 & $\operatorname{Mad} 41$ & hypothetical protein & WP_089310155.1 & 84.88 & Actinomadura mexicana \\
\hline Cluster 1 & Mad 42 & PadR family transcriptional regulator & WP_067474735.1 & 77.20 & Actinomadura hibisca \\
\hline Cluster 1 & $\operatorname{Mad} 43$ & hypothetical protein & WP_131985403.1 & 59.77 & Actinomadura sp. 6K520 \\
\hline Cluster 1 & Mad 44 & hypothetical protein kinase & WP_067640675.1 & 74.19 & Actinomadura latina \\
\hline Cluster 1 & Mad 45 & DEAD/DEAH box helicase & WP_067640681.1 & 93.34 & Actinomadura latina \\
\hline Cluster 1 & $\operatorname{Mad} 46$ & BREX system ATP-binding protein BrxD & WP_067640683.1 & 94.43 & Actinomadura latina \\
\hline Cluster 1 & Mad47 & hypothetical protein & WP_083947146.1 & 83.33 & Actinomadura latina \\
\hline Cluster 1 & $\operatorname{Mad} 48$ & hypothetical protein & WP_028806279.1 & 75.00 & $\begin{array}{l}\text { Streptomyces sp. } \\
\text { 303MFCol5.2 }\end{array}$ \\
\hline Cluster 1 & Mad49 & hypothetical protein & WP_109379674.1 & 70.15 & Streptomyces sp. NWU339 \\
\hline Cluster 1 & $\operatorname{Mad} 50$ & TIGR04222 domain-containing membrane protein & WP_131944619.1 & 89.12 & $\begin{array}{l}\text { Actinomadura } \\
\text { bangladeshensis }\end{array}$ \\
\hline Cluster 1 & Mad51 & hypothetical protein & None & NA & NA \\
\hline Cluster 1 & Mad52 & hypothetical protein & WP_131877791.1 & 78.52 & Actinomadura sp. KC345 \\
\hline Cluster 1 & $\operatorname{Mad53}$ & hypothetical protein & WP_131877789.1 & 58.28 & Actinomadura sp. KC345 \\
\hline Cluster 1 & Mad54 & $\begin{array}{l}\text { helix-turn-helix domain-containing protein } \\
\text { (putative XRE regulator) }\end{array}$ & WP_132147513.1 & 77.78 & Actinomadura sp. 7K507 \\
\hline Cluster 1 & Mad55 & $\begin{array}{l}\text { DUF397 domain-containing protein (putative } \\
\text { abaA regulator) }\end{array}$ & WP_132147510.1 & 73.02 & Actinomadura sp. 7K507 \\
\hline Cluster 1 & Mad56 & $\begin{array}{l}\text { BREX-2 system adenine-specific } \\
\text { DNA-methyltransferase PglX }\end{array}$ & WP_067637150.1 & 67.55 & Actinomadura latina \\
\hline Cluster 1 & $\operatorname{Mad} 57$ & BREX system serine/threonine kinase PglW & WP_067637152.1 & 95.89 & Actinomadura latina \\
\hline Cluster 1 & $\operatorname{Mad} 58$ & $\begin{array}{l}\text { DUF262 domain-containing protein (putative } \\
\text { nuclease) }\end{array}$ & WP_067637154.1 & 78.87 & Actinomadura latina \\
\hline Cluster 1 & $\operatorname{Mad59}$ & CBS domain-containing protein & WP_131900158.1 & 69.14 & Actinomadura sp. $\mathrm{H} 3 \mathrm{C} 3$ \\
\hline Cluster 1 & Mad60 & hydroxybenzoate -AMP ligase & CahJ & 67 & Streptomyces gandocaensis \\
\hline Cluster 1 & Mad61 & aspartate 1-decarboxylase & $\mathrm{CahF}$ & 77 & Streptomyces gandocaensis \\
\hline Cluster 1 & Mad62 & siderophore transporter EntS & WP_132199668.1 & 88.65 & $\begin{array}{l}\text { Actinomadura } \\
\text { darangshiensis }\end{array}$ \\
\hline Cluster 1 & Mad63 & non-ribosomal peptide synthetase & CahA & 58 & Streptomyces gandocaensis \\
\hline Cluster 1 & Mad64 & $\mathrm{Fe}(3+)$-siderophore $\mathrm{ABC}$ transporter permease & CahT3 & 49 & Streptomyces gandocaensis \\
\hline Cluster 1 & Mad65 & $\mathrm{Fe}(3+)$-siderophore $\mathrm{ABC}$ transporter permease & CahT2 & 49 & Streptomyces gandocaensis \\
\hline Cluster 1 & Mad66 & $\begin{array}{l}\text { helix-turn-helix transcriptional regulator } \\
\text { (TetR/AcrR family) }\end{array}$ & WP_089316200.1 & 87.50 & Actinomadura mexicana \\
\hline Cluster 1 & Mad67 & $\mathrm{ABC}$ transporter ATP-binding protein & WP_089316201.1 & 91.00 & Actinomadura mexicana \\
\hline Cluster 1 & Mad68 & $\mathrm{ABC}$ transporter permease & WP_132199663.1 & 83.06 & $\begin{array}{l}\text { Actinomadura } \\
\text { darangshiensis }\end{array}$ \\
\hline Cluster 1 & Mad69 & STAS domain-containing protein & WP_089316203.1 & 83.97 & Actinomadura mexicana \\
\hline Cluster 1 & $\operatorname{Mad} 70$ & hypothetical sensor histidine kinase & WP_089316205.1 & 89.80 & Actinomadura mexicana \\
\hline Cluster 1 & Mad71 & response regulator transcription factor & WP_089316206.1 & 95.09 & Actinomadura mexicana \\
\hline Cluster 1 & Mad72 & hypothetical protein & WP_089316207.1 & 89.33 & Actinomadura mexicana \\
\hline Cluster 1 & $\operatorname{Mad} 73$ & DNA polymerase IV & WP_089316208.1 & 92.37 & Actinomadura mexicana \\
\hline Cluster 1 & Mad74 & cellulose 1,4-beta-cellobiosidase & WP_089316209.1 & 93.32 & Actinomadura mexicana \\
\hline Cluster 1 & Mad75 & $\begin{array}{c}\text { LacI family DNA-binding transcriptional } \\
\text { regulator }\end{array}$ & WP_089316210.1 & 95.65 & Actinomadura mexicana \\
\hline Cluster 1 & Mad76 & cellulose-binding protein & WP_132204372.1 & 87.35 & $\begin{array}{l}\text { Actinomadura } \\
\text { darangshiensis }\end{array}$ \\
\hline Cluster 1 & $\operatorname{Mad} 77$ & cellulose 1,4-beta-cellobiosidase & WP_089316211.1 & 90.24 & Actinomadura mexicana \\
\hline Cluster 1 & $\operatorname{Mad} 78$ & cellulose 1,4-beta-cellobiosidase & WP_089316211.1 & 96.77 & Actinomadura mexicana \\
\hline
\end{tabular}

